TRANSACTIONS OF THE

AMERICAN MATHEMATICAL SOCIETY

Volume 363, Number 4, April 2011, Pages 2211-2247

S 0002-9947(2010)05261-4

Article electronically published on November 16, 2010

\title{
PROX-REGULAR SETS AND EPIGRAPHS IN UNIFORMLY CONVEX BANACH SPACES: VARIOUS REGULARITIES AND OTHER PROPERTIES
}

\author{
FRÉDÉRIC BERNARD, LIONEL THIBAULT, AND NADIA ZLATEVA
}

\begin{abstract}
We continue the study of prox-regular sets that we began in a previous work in the setting of uniformly convex Banach spaces endowed with a norm both uniformly smooth and uniformly convex (e.g., $L^{p}, W^{m, p}$ spaces). We prove normal and tangential regularity properties for these sets, and in particular the equality between Mordukhovich and proximal normal cones. We also compare in this setting the proximal normal cone with different Hölderian normal cones depending on the power types $s, q$ of moduli of smoothness and convexity of the norm. In the case of sets that are epigraphs of functions, we show that $J$-primal lower regular functions have prox-regular epigraphs and we compare these functions with Poliquin's primal lower nice functions depending on the power types $s, q$ of the moduli. The preservation of prox-regularity of the intersection of finitely many sets and of the inverse image is obtained under a calmness assumption. A conical derivative formula for the metric projection mapping of prox-regular sets is also established. Among other results of the paper it is proved that the Attouch-Wets convergence preserves the uniform $r$-prox-regularity property and that the metric projection mapping is in some sense continuous with respect to this convergence for such sets.
\end{abstract}

\section{INTRODUCTION}

Prox-regular sets appear under different names in the literature, depending on the point of view chosen by the authors who often considered them independently. The first one was Federer in [26], where he introduced these sets in $\mathbb{R}^{n}$ as the "sets with positive reach", in order to extend the Steiner polynomial formula to a much larger class of subsets of $\mathbb{R}^{n}$ than those of convex sets or compact $C^{2}$ manifolds. Later, motivated by different purposes, other authors focused their analysis on distinct properties of sets and considered the classes of $p$-convex sets (15]), sets with 2-order tangential property (41]), proximally smooth sets ([18]), prox-regular sets ([39]), and so on. All these concepts are actually known to be the same and to be equivalent in $\mathbb{R}^{n}$ to the notion of positively reached sets. The class of prox-regular sets is much larger than that of convex sets, but it shares with the latter many good properties with regard to the applications in optimization, control theory, etc. and also has rich geometric implications; see, in addition to the works quoted

Received by the editors October 22, 2008 and, in revised form, November 9, 2009.

2010 Mathematics Subject Classification. Primary 49J52, 58C06, 58C20; Secondary 90C30.

Key words and phrases. Distance function, metric projection mapping, uniformly convex Banach space, variational analysis, proximal normal, prox-regular set, epigraph.

The third author was partially supported by the Bulgarian National Fund for Scientific Research, contract DO 02-360/2008.

(c)2010 American Mathematical Society 
above, [17, 42, 33, 24, 34. Such sets are also involved in differential inclusions in mechanics (see, e.g., 19, 24, 42]), in resource allocation mechanisms in economics (see, e.g., [42]), in crowd motion problems (34]), in the theoretical study of viability for differential inclusions subject to constraints (see, e.g., [42]), etc. Concerning related concepts for functions, we refer to [5, 6, 7, 8, 21, 33, 37, 38, 40, 44, and the references therein, and concerning the other similar concept of subsmoothness for sets, we refer to [2].

This paper is based on a previous work of ours 9 where we extended in some sense the study made in [39] of prox-regular sets $C$ in Hilbert spaces. We essentially generalized in [9] the results of [39] in the more general context of uniformly convex Banach spaces, and we provided therein a long list of characterizations including, in particular, one as the Fréchet differentiability of the square distance function $d_{C}^{2}(\cdot)$ around the considered point and another one as the $J$-hypomonotonicity of the proximal normal cone. In the context of Hilbert space the coincidence for a prox-regular set $C$ at $\bar{x}$ between the Mordukhovich normal cone and the proximal normal cone follows directly from the fact (due to the Hilbert structure) that in such a case there exists some nonnegative number $\gamma$ and some neighbourhood $U$ of $\bar{x}$ such that for any proximal normal $x^{*}$ of $C$ at a point $x \in U \cap C$ with $\left\|x^{*}\right\| \leq 1$ one has

$$
\left\langle x^{*}, y-x\right\rangle \leq \gamma\|y-x\|^{2} \quad \text { for all } y \in U \cap C .
$$

The case of a uniformly convex Banach space is not so obvious. Our aim in this paper is on the one hand to show that this important property of equality between the Mordukhovich normal cone and the proximal normal cone still holds for prox-regular sets of uniformly convex Banach spaces and on the other hand to take advantage of this property to provide in the same context several new results including in particular the conical derivative of the metric projection mapping to $C$ and the preservation of prox-regularity under the Attouch-Wets convergence.

In the second section, we give the notation and introduce the main notions needed in the paper. In Section 3, we show the normal and tangential regularity properties of prox-regular sets of a uniformly convex Banach space $X$ and deduce a proximal normal formula for these sets. In Section 4, we prove that the epigraphs of the $J$-primal lower regular functions considered in 9 are prox-regular and compare these functions with Poliquin's primal lower nice functions. In Section 5, we draw a comparison between the prox-regularity concept considered in this paper and taken from [39, 9] and another one from [38, and adapted in [6] to the general Banach context. We also compare the prox-regularity notions when the norm varies in certain families. Section 6 concerns some relationships between different normal cones (of closed sets) related to the moduli of power type of the norm of $X$. Section 7 deals with the preservation of prox-regularity under the intersection and the inverse image. Under a calmness qualification condition, it is shown that the intersection of finitely many prox-regular sets and the inverse image of a prox-regular set by a $C^{1,1}$-mapping inherit the prox-regularity property. In Section 8 we take advantage of the result of tangential regularity of Section 3 to establish a conical derivative formula for the metric projection mapping of prox-regular sets. Finally Section 9 studies the behaviour of the metric projection mapping for a family $\left(C_{t}\right)_{t}$ of uniformly $r$-prox-regular closed sets of $X$ which converges in the sense of AttouchWets to a closed set $C$. It is proved that $C$ inherits the uniform $r$-prox-regularity 
property and that, for any $x_{0}$ with $d\left(x_{0}, C\right)<r$, one has $P_{C_{t}}\left(x_{0}\right) \underset{t}{\longrightarrow} P_{C}\left(x_{0}\right)$, for the metric projection mapping $P_{C}$ onto the closed set $C$.

\section{Notation AND PRELIMINARIES}

Recall that a (real) Banach space $(X,\|\cdot\|)$ is rotund or strictly convex provided that for any $x, y \in X$ with $\|x\|=\|y\|=1$ and $x \neq y$ one has $\left\|\frac{1}{2}(x+y)\right\|<1$. It is when this inequality is uniform in some sense (see below) that the space is said to be uniformly convex. It is known that the strict convexity is equivalent to requiring that, for any nonzero $x, y \in X$ with $x \neq y$, the equality

$$
\|x+y\|=\|x\|+\|y\|
$$

entails $y=\mu x$ for some $\mu>0$ (see, e.g., 22, 25]).

The space $(X,\|\cdot\|)$ is uniformly convex when its modulus of convexity

$$
\delta_{\|\cdot\|}(\varepsilon):=\inf \left\{1-\left\|\frac{x+y}{2}\right\|:\|x\|=\|y\|=1,\|x-y\| \geq \varepsilon\right\}
$$

satisfies $\delta_{\|\cdot\|}(\varepsilon)>0$ for all $\left.\left.\varepsilon \in\right] 0,2\right]$. In such a case the function $\delta_{\|\cdot\|}(\cdot)$ is increasing on $] 0,2]$. The modulus is of power type $q$ if for some real number $k>0$ one has $\delta_{\|\cdot\|}(\varepsilon) \geq k \varepsilon^{q}$ for all $\left.\left.\varepsilon \in\right] 0,2\right]$. By Dvoretzkys's theorem for such a power type $q$ one always has $q \geq 2$.

The space $(X,\|\cdot\|)$ is uniformly smooth if its modulus of smoothness,

$$
\rho_{\|\cdot\|}(\tau):=\sup \left\{\frac{1}{2}\|x+\tau y\|+\frac{1}{2}\|x-\tau y\|-1:\|x\|=1,\|y\|=1\right\} \quad \text { for } \tau \geq 0,
$$

satisfies $\lim _{\tau \downarrow 0} \rho_{\|\cdot\|}(\tau) / \tau=0$. The modulus of smoothness is of power type $s$ with $s>1$ if, for some $c>0$, one has $\rho_{\|\cdot\|}(\tau) \leq c \tau^{s}$ for all $\tau \geq 0$. Dvoretzky's theorem also implies that $s \leq 2$; hence $1<s \leq 2$.

It is possible to renorm any uniformly convex space with an equivalent norm which is both uniformly convex and uniformly smooth. This will always be the context of the paper: Throughout,

$$
\left\{\begin{array}{l}
X \text { is assumed to be a uniformly convex real Banach space endowed with a } \\
\text { norm }\|\cdot\| \text { which is both uniformly convex and uniformly smooth. }
\end{array}\right.
$$

In some statements of the paper, the moduli of uniform convexity and uniform smoothness of the norm $\|\cdot\|$ will be required to be of power type $q$, and of power type $s$, respectively; it will be explicitly emphasized in each such statement. One knows that such a renorm of the uniformly convex space always exists.

The properties of uniformly convex Banach spaces can be found in detail in 23, 3, 22. Let us recall some facts.

It is well known (see, e.g., 23]) that all Hilbert spaces $H$ and the Banach spaces $l^{p}, L^{p}$, and $W_{m}^{p}(1<p<\infty)$ all are (for their usual norms) uniformly convex and uniformly smooth with moduli of convexity and smoothness of power type.

For any real number $\sigma>1$, consider the mapping $J_{\sigma}: X \rightarrow X^{*}$ defined by

$$
J_{\sigma}(x)=\left\{x^{*} \in X^{*}:\left\langle x^{*}, x\right\rangle=\left\|x^{*}\right\| \cdot\|x\|, \quad\left\|x^{*}\right\|=\|x\|^{\sigma-1}\right\} .
$$

For $\sigma=2, J_{2}$ will be simply denoted by $J$ and it is generally called the normalized duality mapping associated with the norm $\|\cdot\|$. As $X$ is reflexive, we have that $J$ is surjective. The mapping $J_{\sigma}$ is the subdifferential of the convex function $\frac{1}{\sigma}\|\cdot\|^{\sigma}$, 
i.e., $J_{\sigma}=\partial\left(\frac{1}{\sigma}\|\cdot\|^{\sigma}\right)$. With the additional uniformly convex property of $X$ and by the choice of the norm that we made, for any $\sigma>1, J_{\sigma}$ is single-valued, bijective and norm-to-norm continuous. The inverse mapping $J^{-1}$ (of $J$ ) will be denoted by $J^{*}$; it is the normalized duality mapping for the dual norm on $X^{*}$. Observe also (by the definitions of $J_{\sigma}$ and $J:=J_{2}$ ) that for $\mathbb{R}_{+}:=[0,+\infty[$,

$$
J_{\sigma}(x) \in \mathbb{R}_{+} J(x) \quad \text { and } \quad J_{\sigma}(t x)=t^{\sigma-1} J_{\sigma}(x) \quad \text { for all } x \in X \text { and } t \geq 0 .
$$

We recall (see [22]) that the mapping $J$ is uniformly continuous over each bounded subset of $X$ (in fact, this property characterizes the uniform smoothness of the norm). Moreover, from [45, (2.16) p. 201 with $p=2$ ], there is a constant $K^{\prime}>0$ such that for all nonzero pairs $(x, y) \in X \times X$,

$$
\langle J(x)-J(y), x-y\rangle \geq K^{\prime}(\max \{\|x\|,\|y\|\})^{2} \delta_{\|\cdot\|}\left(\frac{\|x-y\|}{2 \max \{\|x\|,\|y\|\}}\right)
$$

and by $(3.1)^{\prime}$ of [45, p. 208] one also has some constant $L^{\prime}>0$ such that for all pairs $(x, y) \in X \times X$ with $x \neq y$,

$$
\|J(x)-J(y)\| \leq L^{\prime} \frac{(\max \{\|x\|,\|y\|\})^{2}}{\|x-y\|} \rho_{\|\cdot\|}\left(\frac{\|x-y\|}{\max \{\|x\|,\|y\|\}}\right) .
$$

When the modulus of uniform convexity of the norm $\|\cdot\|$ is of power type $q$, from $\mathrm{Xu}$ and Roach [45, (2.17)' p. 202] again, there exists some constant $K>0$ such that for every $(x, y) \in X \times X$,

$$
\|x+y\|^{q} \geq\|x\|^{q}+q\left\langle J_{q}(x), y\right\rangle+K\|y\|^{q} .
$$

Similarly, whenever the modulus of smoothness of the norm $\|\cdot\|$ is of power type $s$, there exists, according to [45, Remark 5, p. 208], some constant $L>0$ such that, for all $x, y \in X$,

$$
\|x+y\|^{s} \leq\|x\|^{s}+s\left\langle J_{s}(x), y\right\rangle+L\|y\|^{s} .
$$

Observe that (2.4) and (2.5) respectively entail that for all $x, y \in X$,

$$
\left\langle J_{q}(x)-J_{q}(y), x-y\right\rangle \geq \frac{2 K}{q}\|x-y\|^{q}
$$

and

$$
\left\langle J_{s}(x)-J_{s}(y), x-y\right\rangle \leq \frac{2 L}{s}\|x-y\|^{s} .
$$

The inequalities in the lines (2.6) and (2.7) also hold with the normalized duality mapping $J$ in place of $J_{q}$ or $J_{s}$ when they are restricted to bounded subsets of $X$. Indeed, if the norm $\|\cdot\|$ has modulus of convexity (resp. smoothness) of power type $q$ (resp. $s$ ), then for any $r>0$ according to (2.2) (resp. (2.3) ) there exists some positive constant $K_{r}$ (resp. $L_{r}$ ) such that

$$
\langle J(x)-J(y), x-y\rangle \geq K_{r}\|x-y\|^{q} \text { whenever }\|x\| \leq r \text { and }\|y\| \leq r
$$

(respectively

$$
\left.\|J(x)-J(y)\| \leq L_{r}\|x-y\|^{s-1} \text { whenever }\|x\| \leq r \text { and }\|y\| \leq r\right) .
$$

The space $X \times \mathbb{R}$ will be endowed with the norm $\|\cdot\|$ given by $\|(x, r)\|=$ $\sqrt{\|x\|^{2}+r^{2}}$. So, for the normalized duality mapping $J_{X \times \mathbb{R}}: X \times \mathbb{R} \rightarrow X^{*} \times \mathbb{R}$ 
associated with the norm $\|\cdot\|$, one has the equality

$$
J_{X \times \mathbb{R}}(x, r)=(J(x), r) .
$$

When there is no risk of confusion, $J_{X \times \mathbb{R}}$ will be simply denoted by $J$.

We will denote by $\mathbb{B}$ (resp. $\mathbb{B}^{*}$ ) the closed unit ball of $X$ (resp. $X^{*}$ ) and by $B(x, \alpha)$ (resp. $B[x, \alpha]$ ) the open (resp. closed) ball centred at $x$ with radius $\alpha$.

For a closed set $C \subset X$, a nonzero vector $p \in X$ is said to be a primal proximal normal vector to $C$ at $x \in C$ (see [12]) if there are $u \notin C$ and $r>0$ such that $p=r^{-1}(u-x)$ and $\|u-x\|=d_{C}(u)$. (Here $d_{C}(u)$ denotes the distance from $u$ to the set $C$; sometimes it will be convenient to put $d(u, C)$ instead of $\left.d_{C}(u)\right)$. It is known, according to Lau's theorem [31, that in any reflexive Banach space endowed with a Kadec norm, the set of those points which have a nearest point to any fixed closed subset is a dense set. Hence, with the appropriate norm of the uniformly convex space $X$ we are working in, this property holds. Equivalently, a nonzero $p \in X$ is a primal proximal normal vector to $C$ at $x \in C$ if there exists $r>0$ such that $x \in P_{C}(x+r p)$, where $P_{C}$ denotes the metric projection on $C$. We also take by convention the origin of $X$ as a primal normal vector to $C$ at $x$. The cone of all primal proximal normal vectors to $C$ at $x$ will be denoted by $P N_{C}(x)$ and called the primal proximal normal cone of $C$ at $x$. The concept is local in the sense that (see [9, p. 530])

$$
\left\{\begin{array}{l}
\text { for any } u \notin C \text { and any closed ball } V:=B[x, \beta] \text { centred at } x \in C \\
\text { and such that }\|u-x\|=d_{C \cap V}(u), \text { one has } u-x \in P N_{C}(x) .
\end{array}\right.
$$

A continuous linear functional $p^{*} \in X^{*}$ is said to be a proximal normal functional to $C$ at $x \in C$ if $J^{*}\left(p^{*}\right) \in P N_{C}(x)$. This means for $p^{*} \neq 0$ (see [12]) that there are $u \notin C, r>0$ such that $p^{*}=r^{-1} J(u-x)$ and $\|u-x\|=d_{C}(u)$. Or, equivalently, a nonzero $p^{*} \in X^{*}$ is a proximal normal functional to $C$ at $x \in C$ if there exists $r>0$ such that $x \in P_{C}\left(x+r J^{*}\left(p^{*}\right)\right)$. The cone of all proximal normal functionals to $C$ at $x$ will be denoted by $N_{C}^{P}(x)$ or $N^{P}(C ; x)$. One easily verifies that if $p \in P N_{C}(x)$, then $J(p) \in N_{C}^{P}(x)$, and that if $p^{*} \in N_{C}^{P}(x)$, then $J^{*}\left(p^{*}\right) \in P N_{C}(x)$ (keep in mind that $J^{*}=J^{-1}$ is the normalized duality mapping for $X^{*}$ endowed with the dual norm of $\|\cdot\|)$. Hence, $P N_{C}(x)$ and $N_{C}^{P}(x)$ completely determine each other.

We will also need in our development the concept of a Fréchet normal cone $N_{C}^{F}(x)$ or $N^{F}(C ; x)$. A continuous linear functional $x^{*} \in X^{*}$ is said to be a Fréchet normal functional (see [12, 35, 36]) to $C$ at $x \in C$ if for any $\varepsilon>0$ there exists a neighbourhood $U$ of $x$ such that the inequality $\left\langle x^{*}, x^{\prime}-x\right\rangle \leq \varepsilon\left\|x^{\prime}-x\right\|$ holds for all $x^{\prime} \in C \cap U$.

Since the norm of our space $X$ is Fréchet differentiable away from the origin, it is not difficult to verify that for any closed subset $C \subset X$ and any $x \in C$, any proximal normal functional to $C$ at $x$ is also a Fréchet normal functional to $C$ at $x$ (see [12, Corollary 3.1).

We will also use the $\beta$-Hölder normal cone $N_{C}^{\beta}(\cdot)$ or $N^{\beta}(C ; \cdot)$ defined at $x \in C$ by $x^{*} \in N_{C}^{\beta}(x)$ when there exists $\varepsilon, \gamma>0$ such that for any $x^{\prime} \in B(x, \varepsilon) \cap C$, $\left\langle x^{*}, x^{\prime}-x\right\rangle \leq \gamma\left\|x^{\prime}-x\right\|^{\beta}$.

In the context where the norm $\|\cdot\|$ is associated with an inner product $(\cdot \mid \cdot)$, that is, $(X,\|\cdot\|)$ is a Hilbert space, then it is straightforward to verify that $x \in P_{C}(x+r p)$ if and only if

$$
(p \mid y-x) \leq(2 r)^{-1}\|y-x\|^{2} \quad \text { for all } y \in C .
$$


The same description says that for a nonzero vector $p$ there exists some positive $r$ satisfying the above property if and only if there are positive $\varepsilon$ and $\gamma$ such that

$$
\langle J p, y-x\rangle=(p \mid y-x) \leq \gamma\|y-x\|^{2} \quad \text { for all } y \in B(x, \varepsilon) \cap C,
$$

that is, for $\beta=2$ one has $J p \in N_{C}^{\beta}(x)$, and hence $N_{C}^{P}(x)=N_{C}^{\beta}(x)$. Since $N_{C}^{\beta}(x)$ is of course independent of any equivalent norm to $\|\cdot\|$, so is the cone $N_{C}^{P}(x)$ in the Hilbert setting.

The above notions can be translated into the context of functions. Let $f: X \rightarrow$ $\mathbb{R} \cup\{+\infty\}$ be a lower semicontinuous (lsc) function. By definition, the effective domain of $f$ is the set $\operatorname{dom} f:=\{x \in X: f(x)<+\infty\}$ and the epigraph of $f$ is the set epi $f:=\{(x, r) \in X \times \mathbb{R}: f(x) \leq r\}$. Let $x \in \operatorname{dom} f$. We say that $p^{*} \in X^{*}$ is a proximal subgradient of $f$ at $x$ if $\left(p^{*},-1\right)$ is a proximal normal functional to the epigraph of $f$ at $(x, f(x))$. The proximal subdifferential of $f$ at $x$, denoted by $\partial_{P} f(x)$, consists of all such functionals. Thus, we have $p^{*} \in \partial_{P} f(x)$ if and only if $\left(p^{*},-1\right) \in N_{\text {epi } f}^{P}(x, f(x))$. The functional $x^{*} \in X^{*}$ is said to be a Fréchet subgradient of $f$ at $x$ if $\left(x^{*},-1\right)$ is a Fréchet normal functional to the epigraph of $f$ at $(x, f(x))$. The Fréchet subdifferential of $f$ at $x$, denoted by $\partial_{F} f(x)$, consists of all such functionals. If $x \notin \operatorname{dom} f$, then all subdifferentials of $f$ at $x$ are empty, by convention. It is known that for an lsc function $f$ on a reflexive Banach space with a Kadec and Fréchet differentiable norm (in particular, on our above space $X$ ), the set $\operatorname{dom} \partial_{P} f$ is dense in $\operatorname{dom} f$ (see [13, Theorem 7.1). Moreover, from what we saw above, $\partial_{P} f(x) \subset \partial_{F} f(x)$ for all $x \in X$. The Fréchet subgradients are known (see [35]) to have an analytical characterization in the sense that $x \in \partial_{F} f(x)$ if and only if $\liminf _{y \rightarrow x} \frac{f(y)-f(x)-\left\langle x^{*}, y-x\right\rangle}{\|y-x\|} \geq 0$. When $\partial_{F} f(x) \neq \emptyset$, one says that $f$ is Fréchet subdifferentiable at the point $x$. Similarly, a $\beta$-Hölder subgradient of $f$ at $x$ is any functional $x^{*} \in X^{*}$ such that $\left(x^{*},-1\right) \in N_{C}^{\beta}(x, f(x))$. Denoting by $\partial_{\beta} f(x)$ the set of such subgradients, we have $x^{*} \in \partial_{\beta} f(x)$ if there exists $\gamma, \varepsilon>0$ such that for all $y \in B(x, \varepsilon), f(y) \geq f(x)+\left\langle x^{*}, y-x\right\rangle-\gamma\|y-x\|^{\beta}$.

As usual, we will denote by $\psi_{C}$ the indicator function of a closed set $C \subset X$, i.e., $\psi_{C}(y)=0$ if $y \in C$ and $\psi_{C}(y)=+\infty$ otherwise. It is easily checked that $\partial_{P} \psi_{C}(x)=N_{C}^{P}(x)$ for any $x \in C$. The Fréchet and $\beta$-Hölder normal cones of $C$ are related to the indicator function $\psi_{C}$ in a similar way.

The concept of prox-regularity was introduced for functions $f: \mathbb{R}^{n} \rightarrow \mathbb{R} \cup\{+\infty\}$ by Poliquin and Rockafellar in [38, enlarging significantly the class of primal lower nice (pln) functions previously introduced by Poliquin in [37. This class of pln functions considerably generalized the scope of functions that possess as good behaviour as convex functions concerning their regularization, their integrability, their second-order properties, etc.; see [37, 44, 32, 29, 8, 33] and the references therein. The extension of second-order considerations was in fact the main motivation of Poliquin and Rockafellar to defining the class of prox-regular functions, which contains pln functions. They introduced as a special case the concept of prox-regularity of sets. The study of this concept was developed in Hilbert space by Poliquin, Rockafellar and Thibault in [39, where they showed its rich geometric implications. We extended this study in some sense in the context of uniformly convex Banach spaces 
in 9. Taking Definition 2.1 and Proposition 2.2 in 9 into account, we may define the prox-regularity as follows.

Definition 2.1. A closed set $C \subset X$ is called (metrically) prox-regular (with respect to the uniformly convex norm $\|\cdot\|$ ) or $\|\cdot\|$-prox-regular at $\bar{x} \in C$ provided there exist $\varepsilon>0$ and $r>0$ such that for all $x \in C$ and for all $p^{*} \in N_{C}^{P}(x)$ with $\|x-\bar{x}\|<\varepsilon$ and $\left\|p^{*}\right\|<1$ the point $x$ is a nearest point of $\left\{x^{\prime} \in C:\left\|x^{\prime}-\bar{x}\right\|<\varepsilon\right\}$ to $x+r J^{*}\left(p^{*}\right)$.

The metrical aspect is due to the fact that the proximal normal cone $N_{C}^{P}(\cdot)$ is related to the norm $\|\cdot\|$ and depends on it in general when the latter is not a Hilbert norm. Whenever there is no ambiguity concerning either the norm $\|\cdot\|$ or the involvement of the proximal normal cone $N_{C}^{P}(\cdot)$, we will merely say that $C$ is prox-regular at $\bar{x}$.

The crucial fact which needs to be emphasized here is that the real number $r$ of the definition (for which the closed ball $B\left[x+r J^{*}\left(p^{*}\right), r\left\|p^{*}\right\|\right]$ touches the set $C \cap B(\bar{x}, \varepsilon)$ at the point $x$, when $x$ is a boundary point of $C$ with $\|x-\bar{x}\|<\varepsilon$ ) does not depend on either the neighbouring point $x$ or the proximal normal functional $p^{*} \in N_{C}^{P}(x)$ with $\left\|p^{*}\right\|<1$.

We introduced in 9 the following closely related concept, which we called $J$ hypomonotonicity.

Definition 2.2. A set-valued mapping $T: X \rightrightarrows X^{*}$ is said to be $J$-hypomonotone of degree $t \geq 0$ on a subset $U \subset X$ if for any $\left(x_{i}, x_{i}^{*}\right) \in \operatorname{gph} T:=\left\{\left(x, x^{*}\right) \in U \times X^{*}\right.$ : $\left.x^{*} \in T(x)\right\}, i=1,2$, one has

$$
\left\langle J\left[J^{*}\left(x_{1}^{*}\right)-t\left(x_{2}-x_{1}\right)\right]-J\left[J^{*}\left(x_{2}^{*}\right)-t\left(x_{1}-x_{2}\right)\right], x_{2}-x_{1}\right\rangle \leq 0 .
$$

It will be convenient, for any $\delta \geq 0$, to denote below by $N_{C, \delta}^{P}$ the set-valued mapping $N_{C}^{P}$ truncated with $\delta \mathbb{B}^{*}$, i.e.,

$$
N_{C, \delta}^{P}(x):=N_{C}^{P}(x) \cap \delta \mathbb{B}^{*} \text { for all } x .
$$

The following characterizations (and more) were given in 39] in the Hilbert setting and in 9 in the uniformly convex setting.

Theorem 2.3. Assume that the moduli of uniform convexity and uniform smoothness of the norm are of power type. Then a closed set $C \subset X$ is prox-regular at $\bar{x} \in C$ if and only if one of the properties below is fulfilled:

(i) there exist $\varepsilon>0$ and $r>0$ such that for all $x \in C$ and for all $p^{*} \in N_{C}^{P}(x)$ with $\|x-\bar{x}\|<\varepsilon$ and $\left\|p^{*}\right\| \leq 1$,

$$
0 \geq\left\langle J\left[J^{*}\left(p^{*}\right)-r^{-1}\left(x^{\prime}-x\right)\right], x^{\prime}-x\right\rangle, \quad \forall x^{\prime} \in C \text { with }\left\|x^{\prime}-\bar{x}\right\|<\varepsilon ;
$$

(ii) for some $\varepsilon, \tau>0$, the set-valued mapping $N_{C, \varepsilon}^{P}: X \rightrightarrows X^{*}$ is J-hypomonotone of degree $\tau$ on $B(\bar{x}, \varepsilon)$;

(iii) $P_{C}$ is single-valued and norm-to-norm continuous on some neighbourhood $U$ of $\bar{x}$;

(iv) $d_{C}^{2}$ is of class $C^{1}$ on some neighbourhood $U$ of $\bar{x}$;

(v) $d_{C}$ is Fréchet differentiable on $U \backslash C$ for some neighbourhood $U$ of $\bar{x}$.

Anticipating Definition 2.7 below, we see that (i) means that the indicator function $\psi_{C}$ of $C$ is $J$-primal lower regular at $\bar{x}$. Another characterization can be given in terms of the mapping $J_{\sigma}$ in place of the normalized duality mapping $J$. 
Proposition 2.4. Let $\sigma>1$ be any real number. The condition (i) of Theorem 2.3 is equivalent to any one of the following:

$\left(\mathrm{i}_{\sigma}\right)$ there exist $\varepsilon>0$ and $r>0$ such that for all $x \in C$ and all $p^{*} \in N_{C}^{P}(x)$ with $\|x-\bar{x}\|<\varepsilon$ and $\left\|p^{*}\right\| \leq 1$,

$$
0 \geq\left\langle J_{\sigma}\left[J_{\sigma}^{-1}\left(p^{*}\right)-r^{-1}\left(x^{\prime}-x\right)\right], x^{\prime}-x\right\rangle \quad \forall x^{\prime} \in C \text { with }\left\|x^{\prime}-\bar{x}\right\|<\varepsilon ;
$$

$\left(\mathrm{i}_{\sigma}^{\prime}\right)$ there exist $\varepsilon>0$ and $r>0$ such that for all $x \in C, t \geq 0$, and $p^{*} \in N_{C}^{P}(x)$ with $\|x-\bar{x}\|<\varepsilon$ and $\left\|p^{*}\right\| \leq r^{\sigma-1} t^{\sigma-1}$,

$$
0 \geq\left\langle J_{\sigma}\left[J_{\sigma}^{-1}\left(p^{*}\right)-t\left(x^{\prime}-x\right)\right], x^{\prime}-x\right\rangle \quad \forall x^{\prime} \in C \text { with }\left\|x^{\prime}-\bar{x}\right\|<\varepsilon .
$$

Proof. Note first that property (i) of Theorem 2.3 is equivalent to the following property:

for any $x, x^{\prime} \in C \cap B(\bar{x}, \varepsilon)$, any $t \geq 0, p^{*} \in N_{C}^{P}(x)$ with $\left\|p^{*}\right\| \leq r t$,

$$
0 \geq\left\langle J\left[J^{*}\left(p^{*}\right)-t\left(x^{\prime}-x\right)\right], x^{\prime}-x\right\rangle .
$$

Indeed, taking any $t>0, x, x^{\prime} \in C \cap B(\bar{x}, \varepsilon)$ and $p^{*} \in N_{C}^{P}(x)$ with $\left\|p^{*}\right\| \leq r t$ we have by (i),

$$
0 \geq\left\langle J\left[J^{*}\left(r^{-1} t^{-1} p^{*}\right)-r^{-1}\left(x^{\prime}-x\right)\right], x^{\prime}-x\right\rangle,
$$

which is easily seen to be equivalent to (2.13) according to the positive homogeneity of $J$ and $J^{*}$. Further it is obvious that (2.13) still holds for $t=0$. The implication from (i) of Theorem 2.3 to (2.13) is then established. The converse follows from similar arguments.

With this new formulation, we are able to prove that (i) is equivalent to $\left(\mathrm{i}_{\sigma}^{\prime}\right)$.

Suppose that $\left(\mathrm{i}_{\sigma}^{\prime}\right)$ holds. Observe first that for any nonzero $p^{*} \in X^{*}$, one has $J_{\sigma}^{-1}\left(p^{*}\right)=J^{*}\left(\left\|p^{*}\right\|^{\frac{2-\sigma}{\sigma-1}} p^{*}\right)$ and for any nonzero $u \in X$, one has $J_{\sigma}(u)=J\left(\|u\|^{\sigma-2} u\right)$. Hence, putting $\sigma^{\prime}:=\frac{2-\sigma}{\sigma-1}$, for any nonzero $p^{*} \in X^{*}$ and any $t \geq 0$, one has the equivalences

$$
\begin{aligned}
\left\langle J\left[J^{*}\left(p^{*}\right)-t\left(x^{\prime}-x\right)\right], x^{\prime}-x\right\rangle \leq 0 & \Leftrightarrow \\
\left\langle J\left[\left\|p^{*}\right\|^{-\sigma^{\prime}} J_{\sigma}^{-1}\left(p^{*}\right)-t\left(x^{\prime}-x\right)\right], x^{\prime}-x\right\rangle \leq 0 & \Leftrightarrow \\
\left\langle J\left[J_{\sigma}^{-1}\left(p^{*}\right)-t\left\|p^{*}\right\|^{\sigma^{\prime}}\left(x^{\prime}-x\right)\right], x^{\prime}-x\right\rangle \leq 0 & \Leftrightarrow \\
\left\langle J_{\sigma}\left[J_{\sigma}^{-1}\left(p^{*}\right)-t\left\|p^{*}\right\|^{\sigma^{\prime}}\left(x^{\prime}-x\right)\right], x^{\prime}-x\right\rangle \leq 0 . &
\end{aligned}
$$

Now fix any $t>0$, any $x, x^{\prime} \in C \cap B(\bar{x}, \varepsilon)$, and any nonzero $p^{*} \in N_{C}^{P}(x)$ such that $r t \geq\left\|p^{*}\right\|$. The latter inequality ensures that $r t\left\|p^{*}\right\| \sigma^{\prime} \geq\left\|p^{*}\right\|^{\frac{1}{\sigma-1}}$, i.e., $\left\|p^{*}\right\| \leq$ $r^{\sigma-1} t^{\prime \sigma-1}$ for $t^{\prime}:=t\left\|p^{*}\right\| \sigma^{\sigma^{\prime}}$; thus $\left(\mathrm{i}_{\sigma}^{\prime}\right)$ with $t^{\prime}=t\left\|p^{*}\right\|^{\sigma^{\prime}}$ in place of $t$ yields (2.14). By the above equivalences we obtain the inequality (2.13).

Conversely, suppose that (2.13) is fulfilled. Fix any $t \geq 0, x, x^{\prime} \in C \cap B(\bar{x}, \varepsilon)$, and any nonzero $p^{*} \in N_{C}^{P}(x)$ such that $\left\|p^{*}\right\| \leq r^{\sigma-1} t^{\sigma-1}$. One has $\left\|p^{*}\right\|^{\frac{1}{\sigma-1}} \leq r t$ and hence $\left\|p^{*}\right\| \leq r t\left\|p^{*}\right\|^{1-\frac{1}{\sigma-1}}$, which by (2.13), with $t^{\prime}:=t\left\|p^{*}\right\|^{1-\frac{1}{\sigma-1}}=t\left\|p^{*}\right\|^{-\sigma^{\prime}}$ in place of $t$ (where as above $\sigma^{\prime}:=\frac{2-\sigma}{\sigma-1}$ ), yields

$$
0 \geq\left\langle J\left[J^{*}\left(p^{*}\right)-t^{\prime}\left(x^{\prime}-x\right)\right], x^{\prime}-x\right\rangle .
$$

By (2.14) this is equivalent to

$$
0 \geq\left\langle J_{\sigma}\left[J_{\sigma}^{-1}\left(p^{*}\right)-t^{\prime}\left\|p^{*}\right\|^{\sigma^{\prime}}\left(x^{\prime}-x\right)\right], x^{\prime}-x\right\rangle,
$$

which is exactly

$$
0 \geq\left\langle J_{\sigma}\left[J_{\sigma}^{-1}\left(p^{*}\right)-t\left(x^{\prime}-x\right)\right], x^{\prime}-x\right\rangle .
$$

The latter still being true for $p^{*}=0$, we obtain $\left(\mathrm{i}_{\sigma}^{\prime}\right)$. 
The equivalence between $\left(\mathrm{i}_{\sigma}^{\prime}\right)$ and $\left(\mathrm{i}_{\sigma}\right)$ can be argued as in the first part of the proof.

Here we also anticipate Definition 2.7 in calling the property $\left(\mathrm{i}_{\sigma}^{\prime}\right)$ the $J_{\sigma}$-primal lower regularity of $\psi_{C}$.

The concept of prox-regularity in Definition 2.1 is defined as follows from the global point of view.

Definition 2.5. A closed subset $C$ of $X$ is (metrically) uniformly $r$-prox-regular or $r$-uniformly prox-regular if whenever $x \in C$ and $p^{*} \in N_{C}^{P}(x)$ with $\left\|p^{*}\right\|<1$, then $x$ is the unique nearest point of $C$ to $x+r J^{*}\left(p^{*}\right)$.

The next statement uses the open $r$-tube $U_{C}(r)$ around $C$ and the open $r$ neighbourhood $O_{C}(r)$ of $C$ defined by

$$
U_{C}(r):=\left\{x \in X: 0<d_{C}(x)<r\right\} \quad \text { and } \quad O_{C}(r):=\left\{x \in X: d_{C}(x)<r\right\} .
$$

Theorem 2.6 (9]). Assume that the moduli of uniform convexity and uniform smoothness of the norm $\|\cdot\|$ of $X$ are of power type. If the closed set $C \subset X$ is uniformly $r$-prox-regular, then all the assertions below hold. Conversely, (a) or (b) entails that $C$ is uniformly $r$-prox-regular, and (e) entails that $C$ is uniformly $r / 2$-prox-regular.

(a) $d_{C}^{2}$ is of class $C^{1}$ on $O_{C}(r)$.

(b) $d_{C}$ is Fréchet differentiable on $U_{C}(r)$.

(c) $P_{C}$ is single-valued and norm-to-norm continuous on $O_{C}(r)$.

(d) If $u \in O_{C}(r)$ and $x=P_{C}(u)$, then $x \in P_{C}\left(u^{\prime}\right)$ for $u^{\prime}=x+r \frac{u-x}{\|u-x\|}$.

(e) The truncated normal cone mapping $N_{C, r}^{P}$ is $J$-hypomonotone of degree $t$ for any $t \geq 1$.

We were led in 9 to introduce the following $J$-plr concept for functions, which reduces in the Hilbert setting to the Poliquin primal-lower-nice (pln) concept; see [37, 32 and the references in [9].

Definition 2.7. An lsc function $f: X \rightarrow \mathbb{R} \cup\{+\infty\}$ is J-primal lower regular $(J-p l r)$ at $\bar{x} \in \operatorname{dom} f$ if there exist positive constants $\varepsilon, r$ and $\Theta$ such that

$$
f(y) \geq f(x)+\left\langle J\left[J^{*}\left(p^{*}\right)-t(y-x)\right], y-x\right\rangle
$$

for all $x, y \in B(\bar{x}, \varepsilon)$, all $p^{*} \in \partial_{P} f(x)$, and all $t \geq \Theta$ such that $\left\|p^{*}\right\| \leq r t$.

It is easily seen that if $f$ is $J$-plr at $\bar{x}$ with some positive constants $\varepsilon$ and $r$, then it is so for any constants $0<\varepsilon^{\prime} \leq \varepsilon$ and $0<r^{\prime} \leq r$. If the lsc function $f$ is $J$-plr at $\bar{x} \in \operatorname{dom} f$ with positive constants $\varepsilon$ and $r$, then obviously

$$
\left\langle J\left[J^{*}\left(p^{*}\right)-t(y-x)\right]-J\left[J^{*}\left(q^{*}\right)-t(x-y)\right], y-x\right\rangle \leq 0
$$

for all $x, y \in B(\bar{x}, \varepsilon)$, for all $p^{*} \in \partial_{P} f(x), q^{*} \in \partial_{P} f(y)$, and all $t \geq \Theta$ such that $\max \left\{\left\|p^{*}\right\|,\left\|q^{*}\right\|\right\} \leq r t$. This is the analog of the hypomonotonicity of certain truncations of $\partial_{P} f$ that characterizes pln functions in Hilbert spaces; see [37, [32, [8] and the references therein.

Throughout, we will denote by Dom $T$ the domain of the set-valued mapping $T: X \rightrightarrows X^{*}$, i.e., Dom $T:=\{x \in X: T(x) \neq \emptyset\}$. 


\section{Normal and tangential Regularity properties OF PROX-REGULAR SETS}

For a closed set $C \subset X$, the Mordukhovich limiting (or basic) normal cone $N_{C}^{L}(x)$ is defined (see [35]) as the weak* sequential outer limit

$$
N_{C}^{L}(x)={ }^{w^{*} \text {-seq }} \operatorname{Limsup}_{y \rightarrow x} N_{C}^{P}(y):=\left\{w^{*}-\lim x_{n}^{*}: x_{n}^{*} \in N_{C}^{P}\left(x_{n}\right), x_{n} \in C \rightarrow x\right\} .
$$

The following result appears in Ioffe [27, p. 188] with an approximation in $X^{*}$ with respect to the weak star topology, but merely under the local uniform convexity of the norm. For completeness we sketch below how Ioffe's arguments also yield to the following approximation with respect to the strong topology.

Proposition 3.1. Let $C$ be a closed subset of $X$ with $x \in C$ and let $x^{*} \in N_{C}^{F}(x)$. Then for any $\varepsilon>0$ there exist $u_{\varepsilon} \in C$ and $u_{\varepsilon}^{*} \in N_{C}^{P}\left(u_{\varepsilon}\right)$ such that

$$
\left\|u_{\varepsilon}-x\right\|<\varepsilon \quad \text { and } \quad\left\|u_{\varepsilon}^{*}-x^{*}\right\|<\varepsilon \text {. }
$$

In fact, the result uses only the Fréchet differentiability outside of zero of the norm $\|\cdot\|$ and of its dual norm.

Proof. We may suppose that $\left\|x^{*}\right\|_{*}=1$. (For the convenience of the reader, all along the proof the dual norm will be denoted as $\|\cdot\|_{*}$.) By definition there exists some function $\rho$ from $\left[0,+\infty\left[\right.\right.$ into $\left[0,+\infty\left[\right.\right.$ with $\lim _{t \downarrow 0} \rho(t)=0$ and such that

$$
\left\langle x^{*}, y-x\right\rangle \leq \rho(\|y-x\|)\|y-x\| \quad \text { for all } y \in C .
$$

Putting $h:=J^{*} x^{*}$ we have

$$
\left\langle x^{*}, h\right\rangle=\left\|x^{*}\right\|_{*}\|h\|=1 .
$$

Further, by (3.3) we see that $x+t h \notin C$ for positive $t$ small enough. By Lau's theorem for any such $t$ we may choose some $h_{t} \in X$ such that $\left\|h_{t}-h\right\|<t$ and such that the nearest point of $x+t h_{t}$ in $C$ exists, say $u_{t} \in C$. Writing $u_{t}$ in the form $u_{t}=x+t v_{t}$ we have

$$
t\left\|v_{t}-h_{t}\right\|=d_{C}\left(x+t h_{t}\right) \leq t\left\|h_{t}\right\| \quad \text { and hence } \quad\left\|v_{t}\right\| \leq 2\left\|h_{t}\right\| \leq 2(1+t) .
$$

Then taking (3.3) into account we have

$$
\left\langle x^{*}, t v_{t}\right\rangle \leq \rho\left(t\left\|v_{t}\right\|\right)\left\|t v_{t}\right\| \leq 2 t \rho\left(t\left\|v_{t}\right\|\right)(1+t),
$$

which yields

$$
\left\langle x^{*}, v_{t}\right\rangle \leq 2 \rho\left(t\left\|v_{t}\right\|\right)(1+t) .
$$

Now observe by the first inequality of (3.5) that

$$
\left\|h-v_{t}\right\| \leq\left\|h-h_{t}\right\|+\left\|h_{t}-v_{t}\right\| \leq\left\|h-h_{t}\right\|+\left\|h_{t}\right\| \leq\|h\|+2\left\|h-h_{t}\right\|<1+2 t
$$

and hence for $w_{t}:=(1+2 t)^{-1}\left(h-v_{t}\right)$ we have $\left\|w_{t}\right\| \leq 1$. Therefore

$$
(1+2 t)^{-1}\left[1-2 \rho\left(t\left\|v_{t}\right\|\right)(1+t)\right] \leq\left\langle x^{*}, w_{t}\right\rangle \leq 1,
$$

the first inequality being due to (3.4) and (3.6) and the second being due to the inequality $\left\|w_{t}\right\| \leq 1$. Consequently we have

$$
\left\langle x^{*}, w_{t}\right\rangle \rightarrow 1=\left\langle x^{*}, h\right\rangle
$$

and then since the dual norm $\|\cdot\|_{*}$ of $X^{*}$ is Fréchet differentiable at the point $x^{*}$ of the unit sphere of $X^{*}$ and since $\left\|w_{t}\right\| \leq 1$ and $\|h\|=1$, the Šmulian lemma 
(see, e.g., [25, Lemma 8.4]) says that $\left\|w_{t}-h\right\| \rightarrow 0$ as $t \downarrow 0$, which is equivalent to $\left\|v_{t}\right\| \rightarrow 0$.

For positive $t$ sufficiently small, the functional $u_{t}^{*}:=\left\|x+t h_{t}-u_{t}\right\|^{-1} J\left(x+t h_{t}-u_{t}\right)$ is by definition a unit functional in $N_{C}^{P}\left(u_{t}\right)$ and $\left\langle u_{t}^{*}, x+t h_{t}-u_{t}\right\rangle=\left\|x+t h_{t}-u_{t}\right\|$, which is equivalent, by the equality $u_{t}=x+t v_{t}$, to $\left\langle u_{t}^{*}, h_{t}-v_{t}\right\rangle=\left\|h_{t}-v_{t}\right\|$. Since $\left\|v_{t}\right\| \rightarrow 0$ and $h_{t} \rightarrow h$, we derive that $\left\langle u_{t}^{*}, h_{t}\right\rangle \rightarrow\|h\|=1$ and hence

$$
\left\langle u_{t}^{*}, h\right\rangle \rightarrow 1=\left\langle x^{*}, h\right\rangle \text {. }
$$

Remembering that $\left\|u_{t}^{*}\right\|_{*}=1$ and $\left\|x^{*}\right\|_{*}=1$ and that the norm $\|\cdot\|$ is Fréchet differentiable at the point $h$ of the unit sphere of $X$, the Šmulian lemma again entails that $\left\|u_{t}^{*}-x^{*}\right\|_{*} \rightarrow 0$ as $t \downarrow 0$. The proof is then complete because obviously one also has $\left\|u_{t}-x\right\| \rightarrow 0$ as $t \downarrow 0$.

Taking the latter proposition and (3.1) into account, we see (as in Ioffe [27) that the Mordukhovich limiting normal cone above coincides with the (sequential) limiting normal cone obtained as above by replacing $N_{C}^{P}(\cdot)$ with $N_{C}^{F}(\cdot)$, i.e.,

$$
N_{C}^{L}(x)={ }^{w^{*} \text {-seq }} \operatorname{Limsup}_{y \rightarrow x} N_{C}^{F}(y) .
$$

(We must emphasize that one of the important advantages of the expression of $N_{C}^{L}(x)$ in the form of (3.7) is that it makes available the Mordukhovich normal cone in the more general context of Asplund space; see [35, 36]).

We will also need in Section 5 below the strong outer limit

$$
N_{C}^{L, s}(x):=\operatorname{Limsup}_{y \rightarrow x} N_{C}^{P}(y):=\left\{\lim x_{n}^{*}: x_{n}^{*} \in N_{C}^{P}\left(x_{n}\right), x_{n} \in C \rightarrow x\right\}
$$

for $x \in C$. From Proposition 3.1 we also have $N_{C}^{L, s}(x)=\operatorname{Limsup}_{y \rightarrow x} N_{C}^{F}(y)$.

The concept of tangential regularity (see [16]) involved below is related to the Bouligand and Clarke tangent cones. A vector $v$ of $X$ is in the Bouligand tangent cone or Contingent $K_{C}(x)$ to $C$ at $x \in C$ if there exists a sequence of positive numbers $\left(t_{n}\right)_{n}$ converging to 0 and a sequence $\left(v_{n}\right)_{n}$ of $X$ converging to $v$ such that $x+t_{n} v_{n} \in C$ for all $n$. The Clarke tangent cone $T_{C}(x)$ can also be defined in a sequential way. A vector $v \in T_{C}(x)$ provided that for any sequence $\left(x_{n}\right)_{n}$ in $C$ converging to $x$ and for any sequence of positive numbers $\left(t_{n}\right)_{n}$ converging to 0 there exists a sequence $\left(v_{n}\right)_{n}$ of $X$ converging to $v$ with $x_{n}+t_{n} v_{n} \in C$ for all $n$. One always has the inclusion $T_{C}(x) \subset K_{C}(x)$. When the equality holds, one says that the set $C$ is (Clarke or) tangentially regular at $x$.

Through the Clarke tangent cone, the Clarke normal cone $N_{C}^{C l}(x)$ of $C$ at $x \in C$ is defined as the negative polar of the latter; that is,

$$
N_{C}^{C l}(x)=\left\{x^{*} \in X^{*}:\left\langle x^{*}, v\right\rangle \leq 0, \quad \forall v \in T_{C}(x)\right\} .
$$

In any Asplund space (hence in particular in our context), we have (see [35, 36])

$$
N_{C}^{C l}(x)=\overline{c o}^{*}\left({ }^{w^{*}-\operatorname{seq}} \operatorname{Limsup}_{y \rightarrow x} N_{C}^{F}(y)\right),
$$

where $\overline{c o}^{*}$ denotes the weak star closed convex hull.

When the Mordukhovich limiting normal cone of $C$ at $x$ coincides with the Fréchet (resp. proximal) normal cone at $x$, one says that $C$ is normally regular at $x$ with respect to the Fréchet (resp. the proximal) normal cone. Obviously the normal regularity with respect to the proximal normal cone implies the normal regularity with respect to the Fréchet cone (because of the inclusion $N_{C}^{P}(x) \subset N_{C}^{F}(x)$ ). 
We recall (see [14]) that any one of the two above normal regularities entails the (Clarke) tangential regularity. We refer to ([14]) for the development of a detailed comparison between the above concepts of normal and tangential regularities and various others.

The next theorem is one among the results at the heart of the present work.

Theorem 3.2. Assume that the closed set $C$ is prox-regular at $\bar{x} \in C$. Then there exists a neighbourhood $U$ of $\bar{x}$ such that for any $x \in U \cap C$ one has the following normal regularity:

$$
N_{C}^{P}(x)=N_{C}^{F}(x)=N_{C}^{L}(x)=N_{C}^{C l}(x)
$$

and hence

$$
\partial_{P} d_{C}(x)=\partial_{F} d_{C}(x)=\partial_{L} d_{C}(x)=\partial_{C l} d_{C}(x) ;
$$

that is, the distance function itself is subdifferentially regular at all points of $U \cap C$.

$S o$, the set $C$ is, in particular, tangentially regular at $x \in U \cap C$. Further, one has $N_{C}^{\beta}(x) \subset N_{C}^{P}(x)$ for $\beta=2$.

Proof. By assumption, there exist positive real numbers $\varepsilon, r$ with $\varepsilon<1 / 2$ such that for every $x \in C \cap B(\bar{x}, \varepsilon)$ and every $x^{*} \in N_{C}^{P}(x)$ with $\left\|x^{*}\right\| \leq 1$, we have $\left\|x+t J^{*}\left(x^{*}\right)-x^{\prime}\right\| \geq\left\|t J^{*}\left(x^{*}\right)\right\|$ for any $x^{\prime} \in C \cap B(\bar{x}, \varepsilon)$ and $\left.\left.t \in\right] 0, r\right]$. Fix any $x, x^{\prime} \in B(\bar{x}, \varepsilon) \cap C$ and $x^{*} \in N_{C}^{P}(x)$ with $\left\|x^{*}\right\| \leq 1$. Then

$$
\left.\left.\left\|x+t J^{*}\left(x^{*}\right)-x^{\prime}\right\|^{2} \geq\left\|t J^{*}\left(x^{*}\right)\right\|^{2} \quad \text { for any } t \in\right] 0, r\right] .
$$

On the other hand, for any $t \in] 0, r]$, according to the Fréchet differentiability of $\|\cdot\|^{2}$, we have

$$
\begin{aligned}
\left\|x+t J^{*}\left(x^{*}\right)-x^{\prime}\right\|^{2}= & \left\|t J^{*}\left(x^{*}\right)\right\|^{2}+2 \int_{0}^{1}\left\langle J\left[t J^{*}\left(x^{*}\right)+\theta\left(x-x^{\prime}\right)\right], x-x^{\prime}\right\rangle d \theta \\
= & \left\|t J^{*}\left(x^{*}\right)\right\|^{2}+2 t\left\langle x^{*}, x-x^{\prime}\right\rangle \\
& +2 \int_{0}^{1}\left\langle J\left[t J^{*}\left(x^{*}\right)+\theta\left(x-x^{\prime}\right)\right]-J\left[t J^{*}\left(x^{*}\right)\right], x-x^{\prime}\right\rangle d \theta .
\end{aligned}
$$

Combining this with (3.10) we obtain

$$
\left\langle x^{*}, x^{\prime}-x\right\rangle \leq \frac{1}{t}\left\|x^{\prime}-x\right\| \int_{0}^{1}\left\|J\left[t J^{*}\left(x^{*}\right)+\theta\left(x-x^{\prime}\right)\right]-J\left[t J^{*}\left(x^{*}\right)\right]\right\| d \theta .
$$

In the properties of the duality mapping $J$ recalled in Section 2 we saw that $J$ is uniformly continuous over bounded subsets of $X$. Therefore, denoting by $\omega_{r+1}$ the modulus of uniform continuity of $J$ over the bounded set $(r+1) \mathbb{B}$, that is,

$$
\omega_{r+1}(\tau):=\sup \left\{\left\|J(u)-J\left(u^{\prime}\right)\right\|: u, u^{\prime} \in(r+1) \mathbb{B},\left\|u-u^{\prime}\right\| \leq \tau\right\} \quad \text { for } \tau>0,
$$

we have $\omega_{r+1}(\tau) \underset{\tau \downarrow 0}{\longrightarrow} 0$ and (3.11) entails

$$
\left\langle x^{*}, x^{\prime}-x\right\rangle \leq \frac{1}{t}\left\|x^{\prime}-x\right\| \omega_{r+1}\left(\left\|x-x^{\prime}\right\|\right) .
$$

Now fix $x \in C \cap B(\bar{x}, \varepsilon)$ and $x^{*} \in N_{C}^{L}(x)$ and also fix any $\eta>0$. Let $x_{n}^{*} \in N_{C}^{P}\left(x_{n}\right)$ (see (3.1)) such that $x_{n} \in C \underset{n \rightarrow \infty}{\longrightarrow} x$ and $x_{n}^{*} \underset{n \rightarrow \infty}{\stackrel{w^{*}}{\longrightarrow}} x^{*}$. Choose a real number $\gamma>0$ such that $\left\|x_{n}^{*}\right\| \leq \gamma$ for all integers $n$ and choose a positive $\alpha<\varepsilon-\|x-\bar{x}\|$ such that $\omega_{r+1}(\tau) \leq \frac{r \eta}{\gamma}$ for all positive $\tau<\alpha$. Take any $x^{\prime} \in B(x, \alpha) \cap C$. We have $x^{\prime} \in B(\bar{x}, \varepsilon)$ and, for $n$ large enough, $x_{n} \in B(\bar{x}, \varepsilon) \cap C$ and $\left\|x^{\prime}-x_{n}\right\|<\alpha$. By (3.12) for $n$ large enough we then have

$$
\left\langle x_{n}^{*}, x^{\prime}-x_{n}\right\rangle \leq \frac{1}{r}\left\|x_{n}^{*}\right\|\left\|x^{\prime}-x_{n}\right\| \omega_{r+1}\left(\left\|x^{\prime}-x_{n}\right\|\right) \leq \frac{1}{r} \gamma\left\|x^{\prime}-x_{n}\right\| \frac{r \eta}{\gamma}
$$


and hence, passing to the limit for $n \rightarrow \infty$, we obtain

$$
\left\langle x^{*}, x^{\prime}-x\right\rangle \leq \eta\left\|x^{\prime}-x\right\| .
$$

The latter inequality being true for all $x^{\prime} \in B(x, \alpha) \cap C$, this means that $x^{*} \in N_{C}^{F}(x)$. So far we have proved that for any $x \in B(\bar{x}, \varepsilon) \cap C$, we have $N_{C}^{L}(x) \subset N_{C}^{F}(x)$. As the reverse inclusion is also true according to (3.7), we have in fact the equality

$$
N_{C}^{L}(x)=N_{C}^{F}(x) \text { for all } x \in B(\bar{x}, \varepsilon) \cap C .
$$

Moreover, by (3.9), we know that $N_{C}^{C l}(\cdot)=\overline{c o}^{*}\left(N_{C}^{L}\right)(\cdot)$, where $\overline{c o}^{*}$ denotes the weak* closed convex hull in $X^{*}$. Since $N_{C}^{F}(x)$ is convex and (strongly) closed (see 14]), we deduce from the equality (3.13) that we even have

$$
N_{C}^{F}(x)=N_{C}^{L}(x)=N_{C}^{C l}(x) \text { for all } x \in B(\bar{x}, \varepsilon) \cap C .
$$

Let us now prove that the three cones in (3.14) are also equal to the cone of proximal normal functionals to $C$. In our uniformly convex setting where the norm $\|\cdot\|$ of $X$ is uniformly convex and uniformly smooth, we know according to Proposition 3.1 that for any $x \in B(\bar{x}, \varepsilon) \cap C, x^{*} \in N_{C}^{F}(x)$ with $\left\|x^{*}\right\|<1$, there exists $x_{n} \underset{n \rightarrow \infty}{\longrightarrow} x$ with $x_{n} \in C$, and $x_{n}^{*} \in N_{C}^{P}\left(x_{n}\right)$ such that $x_{n}^{*} \underset{n \rightarrow \infty}{\|\|_{3}} x^{*}$. For $n$ large enough we have $x_{n} \in B(\bar{x}, \varepsilon)$ and $\left\|x_{n}^{*}\right\|<1$. For any such integer $n$, for any $\left.\left.t \in\right] 0, r\right]$ and for $x^{\prime} \in B(\bar{x}, \varepsilon) \cap C 1$, we have by (3.10),

$$
\left\|x_{n}+t J^{*}\left(x_{n}^{*}\right)-x^{\prime}\right\|^{2} \geq\left\|t J^{*}\left(x_{n}^{*}\right)\right\|^{2},
$$

which gives, by passing to the limit and by the continuity of $J^{*}$,

$$
\left\|x+t J^{*}\left(x^{*}\right)-x^{\prime}\right\|^{2} \geq\left\|t J^{*}\left(x^{*}\right)\right\|^{2}
$$

i.e., $x^{*} \in N_{C}^{P}(x)$ thanks to the local character (2.11) of the primal proximal normal vector. So for any fixed $x \in B(\bar{x}, \varepsilon) \cap C$ we obtain that $N_{C}^{F}(x) \subset N_{C}^{P}(x) \subset N_{C}^{L}(x)$, which combined with (3.14) gives the equalities

$$
N_{C}^{P}(x)=N_{C}^{F}(x)=N_{C}^{L}(x)=N_{C}^{C l}(x) .
$$

These equalities also ensure that

$$
\partial_{C l} d_{C}(x) \subset N_{C}^{C l}(x) \cap \mathbb{B}^{*}=N_{C}^{P}(x) \cap \mathbb{B}^{*}=\partial_{P} d_{C}(x),
$$

the last equality being due to Proposition 1.2 in [9] (see also [16] for the first inclusion). Consequently, we have

$$
\partial_{P} d_{C}(x)=\partial_{F} d_{C}(x)=\partial_{L} d_{C}(x)=\partial_{C l} d_{C}(x) .
$$

Finally, on the one hand, the equality between $N_{C}^{F}(x)$ and $N_{C}^{C l}(x)$ ensures that $C$ is tangentially regular at $x$ (see [14]), and on the other hand, for $\beta=2$, since one always has $N_{C}^{\beta}(x) \subset N_{C}^{F}(x)$, the inclusion $N_{C}^{\beta}(x) \subset N_{C}^{P}(x)$ follows.

We deduce from Theorem 3.2 a proximal normal formula; Lim sup below is, as in (3.8), the strong outer limit; i.e., for a multivalued mapping $T: X \rightrightarrows X^{*}$,

$$
\underset{\substack{\operatorname{Limsup} \\ y \in D}}{\rightarrow} T(y):=\left\{\lim y_{n}^{*}: y_{n}^{*} \in T\left(y_{n}\right), y_{n} \in D \rightarrow x\right\} .
$$


Proposition 3.3. Assume that the moduli of uniform convexity and of uniform smoothness of the norm $\|\cdot\|$ of $X$ are of power type. If $C$ is prox-regular at $\bar{x} \in X$, then there exists a neighbourhood $U$ of $\bar{x}$ such that for any $x \in U \cap C$,

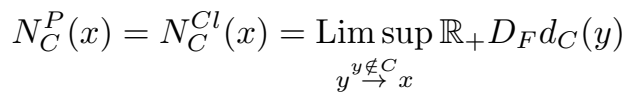

or, equivalently,

$$
\partial_{P} d_{C}(x)=\partial_{C l} d_{C}(x)=[0,1] \operatorname{Limsup}_{y \stackrel{y \notin C}{\rightarrow} x} D_{F} d_{C}(y),
$$

where $D_{F}$ denotes the Fréchet derivative and $\partial_{C l}$ the Clarke subdifferential.

Proof. Remember that the prox-regularity of $C$ entails the Fréchet differentiability

of $d_{C}^{2}$ and the single-valuedness and continuity of $P_{C}$ on an open neighbourhood $U$ of $\bar{x}$; see Theorem 2.3. Fix any $x \in U \cap C$.

Let us prove that

$$
\begin{aligned}
& N_{C}^{P}(x) \subset \operatorname{Limsup} \mathbb{R}_{+} D_{F} d_{C}(y) . \\
& y^{y \notin C} \rightarrow x
\end{aligned}
$$

Recall first that a nonzero continuous linear functional $x^{*}$ is in $N_{C}^{P}(x)$ if and only if there exists $u \in U \backslash C$ such that $x \in P_{C}(u)$ and $x^{*}=\lambda J(u-x)$ for some $\lambda>0$. Then for any $y \in U \cap\{t u+(1-t) x: t \in] 0,1]\}$, one has

$$
J(u-x) \in \mathbb{R}_{+} J(y-x)=\mathbb{R}_{+} D_{F} d_{C}(y)
$$

and thus for $n$ large enough, $x^{*} \in \mathbb{R}_{+} D_{F} d_{C}\left(y_{n}\right)$ with $y_{n}:=x+(u-x) / n$, which proves the desired inclusion.

Now for the reverse inclusion, note that for any $y \in U \backslash C$,

$$
\mathbb{R}_{+} D_{F} d_{C}(y)=\mathbb{R}_{+} J\left(y-P_{C}(y)\right) \subset N_{C}^{P}\left(P_{C}(y)\right) .
$$

Making $y \rightarrow x$, we have by continuity of $P_{C}$ that $P_{C}(y) \rightarrow x$; hence,

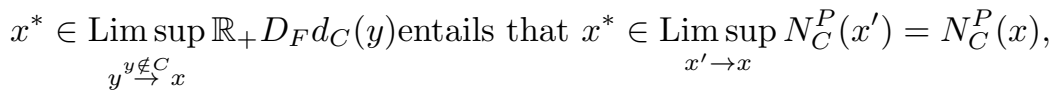

the last equality coming from the normal regularity established in Theorem 3.2, So the reverse inclusion is proved and, of course, by normal regularity with respect to the proximal normal cone (see Theorem 3.2) we obtain $N_{C}^{C l}(x)=N_{C}^{P}(x)$.

The equivalent formulation with the subdifferential of $d_{C}$ comes from the equality $\partial_{P} d_{C}(x)=N_{C}^{P}(x) \cap \mathbb{B}^{*}$ (see [9, Proposition 1.2]) and from the equality $\partial_{P} d_{C}(x)=$ $\partial_{C l} d_{C}(x)$ in Theorem 3.2

Remark 3.4. The proximal normal formula of the above proposition is more precise than the more general one found in Borwein and Giles [10. Theorem 4] for any closed set $C$ in a reflexive Banach space with weaker assumptions on the norm.

\section{EPIGRAPHS OF $J$-PRIMAL LOWER REGULAR FUNCTIONS}

The following proposition and its proof essentially reproduce ideas in [7, Proposition 4.8].

Proposition 4.1. Assume that the moduli of uniform convexity and uniform smoothness of the norm $\|\cdot\|$ of $X$ are of power type. If an lsc function $f$ is $J$-plr at $\bar{x} \in \operatorname{dom} f$, then its epigraph epi $f$ is prox-regular at $(\bar{x}, f(\bar{x}))$. 
We will need the following lemma, given in [17, Exercise 2.1 (d)] in the Hilbert setting. The proof is essentially the same in our general context (without the power types of the norm), but the proximal normal cone is no more identical to $N_{C}^{\beta}(\cdot)$ with $\beta=2$.

Lemma 4.2. For any $x \in \operatorname{dom} f$ and $\alpha>f(x)$, the implication

$$
\left(x^{*}, 0\right) \in N_{\mathrm{epi} f}^{P}(x, \alpha) \Rightarrow\left(x^{*}, 0\right) \in N_{\mathrm{epi} f}^{P}(x, f(x))
$$

holds true.

Proof. Recall that by convention (see Section 2) the norm in $X \times \mathbb{R}$ is defined as $\|(x, r)\|^{2}=\|x\|^{2}+\|r\|^{2}$. Let $\left(x^{*}, 0\right) \in N_{\text {epi } f}^{P}(x, \alpha)$. This means that for all positive $t$ close enough to 0 ,

$$
\inf _{\left(x^{\prime}, \alpha^{\prime}\right) \in \operatorname{epi} f}\left\{\left\|x+t J^{*}\left(x^{*}\right)-x^{\prime}\right\|^{2}+\left(\alpha^{\prime}-\alpha\right)^{2}\right\}=\left\|t J^{*}\left(x^{*}\right)\right\|^{2} .
$$

Choose any $\delta>0$ with $\delta<\alpha-f(x)$. Fix any $\left(x^{\prime}, \alpha^{\prime}\right) \in$ epi $f \cap B((x, f(x)), \delta)$. We have $\alpha^{\prime}-f(x)<\delta<\alpha-f(x)$; hence $\alpha^{\prime}<\alpha$, so $\left(x^{\prime}, \alpha\right) \in$ epi $f$. From (4.1) we obtain

$$
\left\|x+t J^{*}\left(x^{*}\right)-x^{\prime}\right\|^{2}+(\alpha-\alpha)^{2} \geq\left\|t J^{*}\left(x^{*}\right)\right\|^{2},
$$

so

$$
\begin{aligned}
\inf _{\left(x^{\prime}, \alpha^{\prime}\right) \in \operatorname{epi} \mathrm{f} \cap B((x, f(x)), \delta)} & \left\{\left\|x+t J^{*}\left(x^{*}\right)-x^{\prime}\right\|^{2}+\left(\alpha^{\prime}-f(x)\right)^{2}\right\} \\
& \geq \inf _{\left(x^{\prime}, \alpha^{\prime}\right) \in \operatorname{epi}} \inf _{f \cap((x, f(x)), \delta)}\left\{\left\|x+t J^{*}\left(x^{*}\right)-x^{\prime}\right\|^{2}\right\} \geq\left\|t J^{*}\left(x^{*}\right)\right\|^{2},
\end{aligned}
$$

the last inequality being due to (4.2). Since the concept of proximal normal is local (see (2.11) ), we conclude that $\left(x^{*}, 0\right) \in N_{\text {epi } f}^{P}(x, f(x))$.

Proof of the proposition. By the definition of a plr function (see Definition 2.7), there exist $\Theta, \varepsilon, r>0$ such that for any $t \geq \Theta$, any $x \in B(\bar{x}, \varepsilon)$ and $x^{*} \in \partial_{P} f(x)$ with $\left\|x^{*}\right\| \leq r t$,

$$
f(y) \geq f(x)+\left\langle J\left[J^{*}\left(x^{*}\right)-t(y-x)\right], y-x\right\rangle \quad \text { for all } y \in B(\bar{x}, \varepsilon) .
$$

Take $(x, \alpha) \in$ epi $f$ and $\left(x^{*},-\lambda\right) \in N_{\text {epi } f}^{P}(x, \alpha)$ where $\|x-\bar{x}\|<\varepsilon,|\alpha-f(\bar{x})|<\varepsilon$ and $\left\|\left(x^{*},-\lambda\right)\right\| \leq 1$. Fix any $\left(x^{\prime}, \alpha^{\prime}\right) \in$ epi $f$ with $x^{\prime} \in B(\bar{x}, \varepsilon)$.

If $\lambda>0$, then $\alpha=f(x)$ and $\lambda^{-1} x^{*} \in \partial_{P} f(x)$ with $\left\|\lambda^{-1} x^{*}\right\| \leq 1 / \lambda \leq r t$ for every $t \geq \max (\Theta, 1 /(\lambda r))$. Hence by (4.3),

$$
\alpha^{\prime} \geq f\left(x^{\prime}\right) \geq f(x)+\left\langle J\left[J^{*}\left(\lambda^{-1} x^{*}\right)-t\left(x^{\prime}-x\right)\right], x^{\prime}-x\right\rangle,
$$

which entails

$$
0 \geq\left\langle J\left[J^{*}\left(x^{*}\right)-\lambda t\left(x^{\prime}-x\right)\right], x^{\prime}-x\right\rangle-\lambda\left(\alpha^{\prime}-\alpha\right)-\lambda t\left(\alpha^{\prime}-\alpha\right)^{2} .
$$

For any $t^{\prime} \geq \max (\Theta, 1 / r)$, since $t^{\prime} \geq \max (\lambda \Theta, 1 / r)$ because $1 \geq \lambda$, the latter inequality with $t=t^{\prime} / \lambda$, according to (2.10), implies that

$$
0 \geq\left\langle J_{X \times \mathbb{R}}\left[J_{X^{*} \times \mathbb{R}}^{*}\left(x^{*},-\lambda\right)-t^{\prime}\left(\left(x^{\prime}, \alpha^{\prime}\right)-(x, \alpha)\right)\right],\left(x^{\prime}, \alpha^{\prime}\right)-(x, \alpha)\right\rangle .
$$

If $\lambda=0$, we get by Lemma 4.2 that $\left(x^{*}, 0\right) \in N_{\text {epi } f}^{P}(x, f(x))$. Since $N^{P}(\cdot) \subset$ $N^{F}(\cdot)$, by the approximation result in Ioffe [27, p. 190] (see also [35, Lemma 2.37]) there exist sequences $\left(u_{n}, f\left(u_{n}\right)\right) \rightarrow(x, f(x)),\left(u_{n}^{*},-\lambda_{n}\right) \in N_{\text {epi } f}^{F}\left(u_{n}, f\left(u_{n}\right)\right)$ such 
that $\lambda_{n}>0$ and $\left\|\left(u_{n}^{*},-\lambda_{n}\right)-\left(x^{*}, 0\right)\right\| \rightarrow 0$. By Proposition 3.1 for each integer $n$, choose $\left(x_{n}, \alpha_{n}\right) \in \operatorname{epi} f$ and then $\left(y_{n}^{*},-\mu_{n}\right) \in N_{\text {epi } f}^{P}\left(x_{n}, \alpha_{n}\right)$ such that

$$
\left\|\left(u_{n}, f\left(u_{n}\right)\right)-\left(x_{n}, \alpha_{n}\right)\right\|<\lambda_{n} / 2 \text { and }\left\|\left(y_{n}^{*},-\mu_{n}\right)-\left(u_{n}^{*},-\lambda_{n}\right)\right\|<\lambda_{n} / 2 .
$$

The latter inequality ensures in particular that $\mu_{n}>\left(\lambda_{n} / 2\right)>0$ and hence $\alpha_{n}=$ $f\left(x_{n}\right)$. Consequently for $x_{n}^{*}:=\mu_{n}^{-1} y_{n}^{*}$ we have $x_{n}^{*} \in \partial_{P} f\left(x_{n}\right),\left(x_{n}, f\left(x_{n}\right)\right) \rightarrow$ $(x, f(x)), \mu_{n} \downarrow 0$, and $\left\|\mu_{n} x_{n}^{*}-x^{*}\right\| \rightarrow 0$. For $n$ large enough, say $n \geq N$, we have $\left\|x_{n}-\bar{x}\right\|<\varepsilon$ and $\mu_{n}<1$. Suppose for a moment that $x^{*} \neq 0$. Putting

$$
t_{n}:=\max \left(\frac{1}{\mu_{n}} \Theta, \frac{1}{\left\|x^{*}\right\|} \frac{\left\|x_{n}^{*}\right\|}{r}\right)
$$

for $n \geq N$, we see that $t_{n} \geq \max \left(\Theta,\left\|x_{n}^{*}\right\| / r\right)$ since $1 / \mu_{n}>1$ and $1 /\left\|x^{*}\right\| \geq 1$, and hence applying (4.3) with $y=x^{\prime}$ we have

$$
f\left(x^{\prime}\right) \geq f\left(x_{n}\right)+\left\langle J\left[J^{*}\left(x_{n}^{*}\right)-t_{n}\left(x^{\prime}-x_{n}\right)\right], x^{\prime}-x_{n}\right\rangle .
$$

Multiplying this inequality by $\mu_{n}$ and taking the limit, we obtain

$$
0 \geq\left\langle J\left[J^{*}\left(x^{*}\right)-\rho\left(x^{\prime}-x\right)\right], x^{\prime}-x\right\rangle
$$

for $\rho:=\max (\Theta, 1 / r)$. This yields, in particular,

$$
0 \geq\left\langle J\left[J^{*}\left(x^{*}\right)-\rho\left(x^{\prime}-x\right)\right], x^{\prime}-x\right\rangle-\rho\left(\alpha^{\prime}-\alpha\right)^{2} ;
$$

i.e.,

$$
0 \geq\left\langle J_{X \times \mathbb{R}}\left[J_{X^{*} \times \mathbb{R}}^{*}\left(x^{*}, 0\right)-\rho\left(\left(x^{\prime}, \alpha^{\prime}\right)-(x, \alpha)\right)\right],\left(x^{\prime}, \alpha^{\prime}\right)-(x, \alpha)\right\rangle,
$$

and it is obvious that the inequality continues to hold for $x^{*}=0$. Hence both (4.4) and (4.5) hold with $t^{\prime}=\rho$, which entails that the truncated set-valued mapping $N_{\text {epi } f, 1}^{P}$ is $J$-hypomonotone of degree $\rho$ on $B\left(\bar{x}, \varepsilon^{\prime}\right)$ and hence by Theorem 2.3, the set epi $f$ is prox-regular at $(\bar{x}, f(\bar{x}))$.

Remembering that the Clarke subdifferential operator $\partial_{C l}$ and the Mordukhovich limiting subdifferential $\partial_{L}$ satisfy, for any lsc function $f$ (see [16, 35]),

$$
\partial_{C l} f(x)=\left\{x^{*}:\left(x^{*},-1\right) \in N_{\mathrm{epi} f}^{C l}(x, f(x))\right\}
$$

and

$$
\partial_{L} f(x)=\left\{x^{*}:\left(x^{*},-1\right) \in N_{\text {epi } f}^{L}(x, f(x))\right\},
$$

from Theorem 3.2 we have the following corollary.

Corollary 4.3. Assume that the moduli of uniform convexity and smoothness of the norm $\|\cdot\|$ of $X$ are of power type. If $f$ is $J$-plr at $\bar{x}$, then there exists a neighbourhood $U$ of $\bar{x}$ such that for $\beta=2$ one has

$$
\partial_{\beta} f(x) \subset \partial_{P} f(x)=\partial_{F} f(x)=\partial_{L} f(x)=\partial_{C l} f(x) \quad \text { for all } x \in U .
$$

By considering the following property of $f$ being uniformly $J$-plr, we obtain similarly the uniform prox-regularity of epi $f$. The function $f$ is uniformly $J$-plr on $X$ provided that there exist positive constants $\Theta, r$ such that for any $t \geq \Theta$, any $x \in X$ and $x^{*} \in \partial_{P} f(x)$ with $\left\|x^{*}\right\| \leq r t$,

$$
f(y) \geq f(x)+\left\langle J\left[J^{*}\left(x^{*}\right)-t(y-x)\right], y-x\right\rangle \quad \text { for all } y \in X .
$$

Proposition 4.4. Assume that the moduli of uniform convexity and smoothness of the norm $\|\cdot\|$ of $X$ are of power type. If $f$ is uniformly J-plr on $X$ with parameter $r, \Theta$, then epi $f$ is uniformly $r^{\prime}$-prox-regular for some $r^{\prime} \geq \frac{1}{2} \min (1 / \Theta, r)$. 
Proof. According to the proof of Proposition 4.1, the truncated set-valued mapping $N_{\text {epi } f, 1}^{P}$ is $J$-hypomonotone of degree $\rho=\max (\Theta, 1 / r)$. With $\delta=\rho^{-1}$, that fact is equivalent to $N_{\text {epi } f, \delta}^{P}$ being $J$-hypomonotone of degree 1, which by Theorem 2.6 (e) allows us to conclude the proof.

That proposition allows us to establish the $J$-plr property of the basic function equal to the opposite of the square of the norm whenever the latter has moduli of convexity and smoothness of power type.

Proposition 4.5. Assume that the moduli of uniform convexity and smoothness of the norm $\|\cdot\|$ of $X$ are of power type. Then the opposite function of the square of the norm, say $-\|\cdot\|^{2}$, is uniformly $J$-plr on $X$ and hence, in particular, epi $\left(-\|\cdot\|^{2}\right)$ is uniformly $r$-prox-regular for some $r \geq \frac{1}{4}$.

Proof. For any $x \in X, y=x+h \in X, x^{*}=\partial\left(-\|\cdot\|^{2}\right)(x)=-2 J(x)$, by the convexity of $\|\cdot\|^{2}$, we may write

$$
\|x\|^{2}=\|y-h\|^{2} \geq\|y\|^{2}+\langle 2 J(y),-h\rangle .
$$

So, we have

$$
-\|y\|^{2} \geq-\|x\|^{2}+\langle-2 J(y), h\rangle,
$$

which yields, according to the equality $-2 y=J^{*}\left(x^{*}\right)-2(y-x)$, that

$$
-\|y\|^{2} \geq-\|x\|^{2}+\left\langle J\left[J^{*}\left(x^{*}\right)-2(y-x)\right], y-x\right\rangle .
$$

This entails the $J$-hypomonotonicity of degree 2 of $N_{C, 1}^{P}$ where $C:=\operatorname{epi}\left(-\|\cdot\|^{2}\right)$; hence the conclusion follows from (e) of Theorem 2.6 and Proposition 4.4.

In order to compare $J$-plr functions with pln functions, we suppose in Proposition 4.6 and Corollary 4.7 below that the modulus of uniform convexity is of power type $q=2$. Let us recall the definition of pln functions, introduced by Poliquin in $\mathbb{R}^{n}$, studied in the Hilbert setting with further developments in [32. In [6], the definition was slightly extended in a Banach space setting into a $\partial$-pln concept a priori depending on the choice of a subdifferential operator $\partial$. This is the definition given below. See also [29, 8] concerning pln functions.

Following [20] (see also [6] and the references therein), a subdifferential operator $\partial$ associates with each function $f: X \rightarrow \mathbb{R} \cup\{+\infty\}$ a multivalued mapping $\partial f: X \rightrightarrows X^{*}$ and satisfies various assumptions commonly fulfilled by the usual subdifferentials (Fréchet, etc.) on appropriate spaces. Here we will just assume that for any function $f$ from $X$ into $\mathbb{R} \cup\{+\infty\}$, one has

$$
\partial_{P} f(x) \subset \partial f(x) \text { for all } x \in X \text {. }
$$

An lsc function $f$ is $\partial$-pln at $\bar{x} \in \operatorname{dom} f$ if there are $\varepsilon, c, \Theta>0$ such that whenever $t \geq \Theta, x^{*} \in \partial f(x)$ with $\|x-\bar{x}\|<\varepsilon$ and $\left\|x^{*}\right\| \leq c t$, one has

$$
f\left(x^{\prime}\right) \geq f(x)+\left\langle x^{*}, x^{\prime}-x\right\rangle-\frac{t}{2}\left\|x^{\prime}-x\right\|^{2} \text { for all } x^{\prime} \in B(\bar{x}, \varepsilon) .
$$

Obviously, for $\beta=2$ one has $\partial f(\cdot) \subset \partial_{\beta} f(\cdot)$ on $B(\bar{x}, \varepsilon)$ for such a function $f$.

Note that examples of subdifferential operators that contain the proximal subdifferential operator $\partial_{P}$ are given by $\partial_{P}$ itself, by $\partial_{F}$, and hence also by any of the many subdifferential operators that contain $\partial_{F}$.

Proposition 4.6. Assume that the modulus of uniform convexity of the norm $\|\cdot\|$ of $X$ is of power type $q=2$. If $f$ is $\partial$-pln at $\bar{x}$, then $f$ is $J$-plr at $\bar{x}$. 
Proof. Under the assumptions, there exist positive numbers $\varepsilon, r, \Theta$ such that for any $\left(x, x^{*}\right) \in \operatorname{gph} \partial_{P} f$ with $\|x-\bar{x}\|<\varepsilon,\left\|x^{*}\right\| \leq r t$ and $t \geq \Theta$,

$$
f\left(x^{\prime}\right) \geq f(x)+\left\langle x^{*}, x^{\prime}-x\right\rangle-\frac{t}{2}\left\|x^{\prime}-x\right\|^{2} \text { for all } x^{\prime} \in B(\bar{x}, \varepsilon) .
$$

Further, for any $x^{*} \in X^{*}, h \in X, t^{\prime}>0$ we may write

$$
\begin{aligned}
\left\langle x^{*}, h\right\rangle & =\left\langle J\left[J^{*}\left(x^{*}\right)\right]-J\left[J^{*}\left(x^{*}\right)-t^{\prime} h\right], h\right\rangle+\left\langle J\left[J^{*}\left(x^{*}\right)-t^{\prime} h\right], h\right\rangle \\
& \geq \frac{K}{t^{\prime}}\left\|t^{\prime} h\right\|^{2}+\left\langle J\left[J^{*}\left(x^{*}\right)-t^{\prime} h\right], h\right\rangle,
\end{aligned}
$$

the inequality being due to (3.7) with $q=2$. Hence taking $h=x^{\prime}-x$, we have for any $t^{\prime}>0$,

$$
\left\langle x^{*}, x^{\prime}-x\right\rangle-K t^{\prime}\left\|x^{\prime}-x\right\|^{2} \geq\left\langle J\left[J^{*}\left(x^{*}\right)-t^{\prime}\left(x^{\prime}-x\right)\right], x^{\prime}-x\right\rangle .
$$

Combining the latter inequality and (4.7), we see that for any $t^{\prime} \geq \Theta /(2 K),\left(x, x^{*}\right) \in$ gph $\partial_{P} f$ with $\|x-\bar{x}\|<\varepsilon$ and $\left\|x^{*}\right\| \leq(2 K r) t^{\prime}$, we have

$$
f\left(x^{\prime}\right) \geq f(x)+\left\langle J\left[J^{*}\left(x^{*}\right)-t^{\prime}\left(x^{\prime}-x\right)\right], x^{\prime}-x\right\rangle \quad \text { for all } x^{\prime} \in B(\bar{x}, \varepsilon) ;
$$

that is, $f$ is $J$-plr with parameters $\varepsilon, 2 r K, \Theta /(2 K)$.

Corollary 4.7. Assume that the moduli of uniform convexity and smoothness of the norm $\|\cdot\|$ of $X$ are of power type. Under the inclusion $\partial f(\cdot) \subset \partial_{C l} f(\cdot)$ and the assumption of Proposition 4.6, the lsc function $f$ is $\partial-p l n$ at $\bar{x}$ if and only if $f$ is $\partial_{P}-p l n$ at $\bar{x}$ and then for $\beta=2$ one has

$$
\partial_{\beta} f(x)=\partial_{P} f(x)=\partial f(x)=\partial_{F} f(x)=\partial_{L} f(x)=\partial_{C l} f(x)
$$

for all $x$ in a neighbourhood of $\bar{x}$.

Proof. Suppose that $f$ is $\partial$-pln at $\bar{x}$. On the one hand, by (4.6), it is $\partial_{P}$-pln at $\bar{x}$. On the other hand, by the definition of the $\partial$-pln property, the inclusion $\partial f(x) \subset \partial_{\beta} f(x)$ holds for $\beta=2$ and for $x$ in a neighbourhood of $\bar{x}$, and by Proposition 4.6, the function $f$ is also $J$-plr at $\bar{x}$. Therefore, using (4.6) and Corollary 4.3. we obtain the equalities of the corollary.

Conversely, if $f$ is $\partial_{P}$-pln at $\bar{x}$, then Proposition 4.6 again entails that $f$ is $J$-plr at $\bar{x}$ and hence by Proposition 4.1 and Theorem 3.2 we have $\partial_{P} f(x)=\partial_{C l} f(x)$ for all $x$ in some neighbourhood of $\bar{x}$. Combining the latter equality with (4.6) and the inclusion assumption $\partial f(\cdot) \subset \partial_{C l} f(\cdot)$ we obtain that $\partial f(x)=\partial_{P} f(x)$ for all $x$ near $\bar{x}$. So $f$ is $\partial$-pln at $\bar{x}$.

We have a symmetrical result when the modulus of smoothness is $s=2$.

Proposition 4.8. Assume that the moduli of uniform convexity and uniform smoothness of the norm $\|\cdot\|$ are of power type and that the power type of smoothness is $s=2$. If $f$ is $J$-plr at $\bar{x}$, then $f$ is $\partial$-pln at $\bar{x}$ for any subdifferential $\partial$ satisfying the inclusions $\partial_{P} f(\cdot) \subset \partial f(\cdot) \subset \partial_{C l} f(\cdot)$.

Proof. By Corollary 4.3, for all $x$ near $\bar{x}$ we have $\partial_{C l} f(x)=\partial_{P} f(x)$ and hence $\partial_{P} f(x)=\partial f(x)$. The $J$-plr assumption then entails that there exist positive numbers $\varepsilon, r, \Theta$ such that for any $t \geq \Theta,\left(x, x^{*}\right) \in \operatorname{gph} \partial_{P} f$ with $\|x-\bar{x}\|<\varepsilon,\left\|x^{*}\right\| \leq r t$,

$$
f\left(x^{\prime}\right) \geq f(x)+\left\langle J\left[J^{*}\left(x^{*}\right)-t\left(x^{\prime}-x\right)\right], x^{\prime}-x\right\rangle \text { for all } x^{\prime} \in B(\bar{x}, \varepsilon) .
$$


Now fix any $t \geq \Theta, x^{\prime}, x \in B(\bar{x}, \varepsilon)$ and $x^{*} \in \partial f(x)$ with $\left\|x^{*}\right\| \leq r t$. By (2.7) since $s=2$ (the power type of smoothness of the norm), we have

$$
\left\langle J\left[J^{*}\left(x^{*}\right)-t\left(x^{\prime}-x\right)\right]-J\left[J^{*}\left(x^{*}\right)\right],-t\left(x^{\prime}-x\right)\right\rangle \leq L\left\|t\left(x^{\prime}-x\right)\right\|^{2},
$$

which is equivalent to

$$
\left\langle J\left[J^{*}\left(x^{*}\right)-t\left(x^{\prime}-x\right)\right], x^{\prime}-x\right\rangle \geq\left\langle x^{*}, x^{\prime}-x\right\rangle-t L\left\|x^{\prime}-x\right\|^{2} .
$$

Then according to (4.8) we obtain

$$
f\left(x^{\prime}\right)-f(x) \geq\left\langle x^{*}, x^{\prime}-x\right\rangle-t L\left\|x^{\prime}-x\right\|^{2} .
$$

So $f$ is $\partial$-pln at $\bar{x}$.

Corollary 4.9. Assume that the moduli of uniform convexity and smoothness of the norm $\|\cdot\|$ of $X$ are of power type and that the power type of smoothness is $s=2$. Under the assumption of Proposition 4.8, if the lsc function $f$ is $J$-plr at $\bar{x}$, then for $\beta=2$,

$$
\partial_{\beta} f(x)=\partial_{P} f(x)=\partial f(x)=\partial_{F} f(x)=\partial_{L} f(x)=\partial_{C l} f(x)
$$

for all $x$ in a neighbourhood of $\bar{x}$.

Proof. This a consequence of Corollary 4.3 and Proposition 4.8

Uniformly pln functions are defined analogously to uniformly plr functions; i.e., $f$ is uniformly $\partial$-pln if there are $c, \Theta>0$ such that whenever $t \geq \Theta, x^{*} \in \partial f(x)$ with $\left\|x^{*}\right\| \leq c t$,

$$
f\left(x^{\prime}\right) \geq f(x)+\left\langle x^{*}, x^{\prime}-x\right\rangle-\frac{t}{2}\left\|x^{\prime}-x\right\|^{2} \text { for all } x^{\prime} \in X .
$$

The two previous results proved above for $\partial$-pln functions at a point $\bar{x}$ of $X$ also hold for uniformly $\partial$-pln functions: just replace " $\partial$-pln (resp. $J$-plr) at $\bar{x}$ " with "uniformly $\partial$-pln (resp. uniformly $J$-plr)".

\section{Prox-Regularity and $N$-hyporegularity}

Let $N(\cdot)$ be a given normal cone concept (e.g., $N^{F}(\cdot), N^{P}(\cdot)$, etc.) associated with a subdifferential operator $\partial$; i.e., for any closed set $C$ with indicator function $\psi_{C}$, one has $N_{C}=\partial \psi_{C}$ and $\partial f(x)=\left\{x^{*} \in X^{*}:\left(x^{*},-1\right) \in N_{\text {epi } f}(x, f(x))\right\}$. The normal cone $N(\cdot)$ is assumed to satisfy, for any closed set $C$, the inclusion $N_{C}^{P}(\cdot) \subset N_{C}(\cdot)$. Following [38] and the adaptation in [6], the set $C \subset X$ is $N$ hyporegular at $\bar{x} \in C$ if there exist $\varepsilon, r>0$ such that for any $x^{*} \in N_{C}(x)$ with $\|x-\bar{x}\|<\varepsilon,\left\|x^{*}\right\| \leq 1$,

$$
0 \geq\left\langle x^{*}, x^{\prime}-x\right\rangle-\frac{1}{2 r}\left\|x^{\prime}-x\right\|^{2} \text { for all } x^{\prime} \in B(\bar{x}, \varepsilon) \cap C .
$$

Of course, (5.1) holds if and only if the truncated normal cone $N_{C}(\cdot) \cap \mathbb{B}^{*}$ is hypomonotone near $\bar{x}$ in the usual sense, that is, there exist some $\varepsilon, r>0$ such that for all $x_{i} \in C \cap B(\bar{x}, \varepsilon)$ and $x_{i}^{*} \in N_{C}\left(x_{i}\right) \cap \mathbb{B}^{*}, i=1,2$ one has

$$
\left\langle x_{1}^{*}-x_{2}^{*}, x_{1}-x_{2}\right\rangle \geq-\frac{1}{r}\left\|x_{1}-x_{2}\right\|^{2} .
$$

In [6] a set $C$ satisfying (5.1) with $N(\cdot)$ has been called prox-regular with respect to the normal cone $N(\cdot)$. Here we prefer to use the name of $N$-hyporegular set because of the characterization (5.2) as the hypomonotonicity of $N_{C}(\cdot) \cap \mathbb{B}^{*}$ (which has nothing to do with the metric projection mapping) and to reserve the name 
of prox-regularity set merely to translate the regularity property of the metric projection mapping in Definition 2.1 or in (iii) of Theorem 2.3.

We observe by (5.1) that $C$ is $N$-hyporegular at $\bar{x}$ if and only if its indicator function $\psi_{C}$ is $\partial$-pln at $\bar{x}$. Remember (see Theorem 2.3 (i)) that $C$ is prox-regular at $\bar{x}$ if and only if $\psi_{C}$ is $J$-plr at $\bar{x}$. Similarly, the uniform $N$-hyporegularity means that the inequality (5.1) holds for all $x, x^{\prime} \in C$ and $x^{*} \in N_{C}(x) \cap \mathbb{B}^{*}$ and this corresponds to the uniform $\partial$-pln property for the indicator function $\psi_{C}$. In the same way, the uniform prox-regularity of $C$ is equivalent to the uniform $J$-plr property of the function $\psi_{C}$. Remember that $q$ and $s$ are the moduli of convexity and of smoothness respectively, and that they satisfy $1<s \leq 2 \leq q$. The case $q=2$ corresponds for instance to $L^{p}$ spaces with $\left.\left.p \in\right] 1,2\right]$ and $s=2$ to $L^{p}$ spaces with $p \in[2,+\infty[$.

In the case $q=2$, we have from the previous section the following result.

Corollary 5.1. Assume that the moduli of uniform convexity and smoothness of the norm $\|\cdot\|$ of $X$ are of power type. When the power type of the modulus of convexity is $q=2$, a closed set $C \subset X$ is $N$-hyporegular at $\bar{x} \in C$ (resp. uniformly $N$-hyporegular) for a normal cone $N$ which satisfies $N^{P}(\cdot) \subset N(\cdot) \subset N^{C l}(\cdot)$ if and only if it is $N^{P}$-hyporegular at $\bar{x}$ (resp. uniformly $N^{P}$-hyporegular), and then it is also prox-regular at $\bar{x}$ (resp. uniformly prox-regular) and for $\beta=2$ one has $N_{C}^{\beta}(x)=N_{C}^{P}(x)=N_{C}(x)=N_{C}^{C l}(x)$ for any $x$ in some neighbourhood of $\bar{x}$ (resp. any $x \in C)$.

Proof. The corollary is a direct consequence of Proposition 4.6] and Corollary 4.7.

In the case $s=2$ we have the reverse implication. If the moduli of uniform convexity and smoothness are of power type with the power type of smoothness $s=2$, then its proof directly follows from Proposition 4.8 and Corollary 4.9. In fact the proof below shows that the result holds without requiring that the modulus of uniform convexity be of power type.

Proposition 5.2. Assume that the modulus of smoothness of the norm $\|\cdot\|$ of $X$ is of power type $s=2$. If a closed set $C \subset X$ is prox-regular at $\bar{x} \in C$ (resp. uniformly prox-regular), then it is $N$-hyporegular at $\bar{x}$ (resp. uniformly $N$-hyporegular) for any normal cone $N$ which satisfies $N^{P}(\cdot) \subset N(\cdot) \subset N^{C l}(\cdot)$, and further for $\beta=2$ one has

$$
N_{C}^{\beta}(x)=N_{C}^{P}(x)=N_{C}(x)=N_{C}^{C l}(x)
$$

for any $x$ in some neighbourhood of $\bar{x}$ (resp. any $x \in C$ ).

Proof. We prove the result when we have local prox-regularity at a point.

The prox-regularity assumption entails by Proposition 2.2 in 9 that there exist positive numbers $\varepsilon, r$ such that for any $x \in B(\bar{x}, \varepsilon) \cap C$ and for any $x^{*} \in N_{C}^{P}(x)$ with $\left\|x^{*}\right\| \leq 1$,

$$
0 \geq\left\langle J\left[J^{*}\left(x^{*}\right)-r^{-1}\left(x^{\prime}-x\right)\right], x^{\prime}-x\right\rangle, \quad \forall x^{\prime} \in C \text { with }\left\|x^{\prime}-\bar{x}\right\|<\varepsilon .
$$

Now fix any $x^{\prime}, x \in C \cap B(\bar{x}, \varepsilon)$ and $x^{*} \in N_{C}^{P}(x)$ with $\left\|x^{*}\right\| \leq 1$. By (2.7) since $s=2$ (the power type of smoothness of the norm), we have

$$
\left\langle J\left[J^{*}\left(x^{*}\right)-r^{-1}\left(x^{\prime}-x\right)\right]-J\left[J^{*}\left(x^{*}\right)\right],-r^{-1}\left(x^{\prime}-x\right)\right\rangle \leq L\left\|r^{-1}\left(x^{\prime}-x\right)\right\|^{2},
$$


which is equivalent to

$$
\left\langle J\left[J^{*}\left(x^{*}\right)-r^{-1}\left(x^{\prime}-x\right)\right], x^{\prime}-x\right\rangle \geq\left\langle x^{*}, x^{\prime}-x\right\rangle-L r^{-1}\left\|x^{\prime}-x\right\|^{2} .
$$

Then according to (5.3) we obtain

$$
0 \geq\left\langle x^{*}, x^{\prime}-x\right\rangle-L r^{-1}\left\|x^{\prime}-x\right\|^{2} .
$$

So the set $C$ is also $N^{P}$-hyporegular at $\bar{x}$.

Further, putting $\beta=2$, on the one hand the $N^{P}$-hyporegularity ensures us that for an appropriate neighbourhood $U$ of $\bar{x}$, for any $x \in U \cap C$, the inclusion $N_{C}^{P}(x) \subset N_{C}^{\beta}(x)$ holds, and on the other hand by Theorem 3.2, we know that $N_{C}^{\beta}(x) \subset N_{C}^{P}(x)=N_{C}^{C l}(x)$. Since by assumption we have the inclusions $N_{C}^{P}(\cdot) \subset$ $N_{C}(\cdot) \subset N_{C}^{C l}(\cdot)$, we obtain that for $\beta=2$,

$$
N_{C}^{\beta}(x)=N_{C}^{P}(x)=N_{C}(x)=N_{C}^{C l}(x) \text { for all } x \in U \cap C
$$

and consequently, $C$ is also $N$-hyporegular.

For a family of norms $\left(\|\cdot\|_{i}\right)_{i \in I}$, let us denote by $N_{i}^{\text {? }}$ the normal cone $N^{\text {? }}$ (e.g., $N^{F}, N^{C l}, N^{P}$, etc.) obtained by using the norm $\|\cdot\|_{i}$ in its definition and computation. The $N_{i}^{P}$ cones for a closed set $C$ will be denoted by $N_{i}^{P}(C ; \cdot)$. When the norms $\|\cdot\|_{i}$ and $\|\cdot\|_{j}$ are equivalent, some normal cone concepts $N^{?}$ yield $N_{i}^{?}=N_{j}^{?}$, as in the cases $N^{?}=N^{F}, N^{\beta}, N^{L, s}, N^{L}, N^{C l}$ for instance. We will speak of $\left(\|\cdot\|_{i}, N\right)$-hyporegularity of a set $C$ when the property (5.1) is satisfied with the norm $\|\cdot\|=\|\cdot\|_{i}$ and with the cone $N=N_{i}^{?}$. When $C$ is $\left(\|\cdot\|_{i}, N^{?}\right)$ hyporegular for any $i \in I$, we will say that $C$ is $N^{\text {? }}$-hyporegular relative to the family $\left(\|\cdot\|_{i}\right)_{i \in I}$. Similarly, the concept of prox-regularity in Definition 2.1 is a priori norm dependent, so for a given norm $\|\cdot\|$ it has been said in that definition that the set $C$ is $\|\cdot\|$-prox-regular.

From now on in the remainder of this section we address the problem of comparing the $\left(\|\cdot\|_{i}, N^{?}\right)$-hyporegularities and $\|\cdot\|_{i}$-prox-regularities of a set $C$ for norms in a given family $\left(\|\cdot\|_{i}\right)_{i \in I}$, and of comparing accordingly the normal cones $N_{i}^{P}(C ;$.$) for such a set C$.

Note first that by passing to the limit in (5.1) and by using the comments preceding and following Proposition 3.1, it is easily seen, for a norm $\|\cdot\|$ which is both uniformly convex and uniformly smooth, that whenever $C$ is $\left(\|\cdot\|, N^{\text {? }}\right)$ hyporegular at a point $\bar{x}$ with $N^{?}=N^{P}$ or $N^{?}=N^{F}$, then it is $\left(\|\cdot\|, N^{L, s}\right)$ hyporegular at $\bar{x}$ with the same parameters. Further for some neighbourhood $U$ of $\bar{x}$ and for $\beta=2$ the inclusion $N_{C}^{L}(x) \subset N_{C}^{\beta}(x)$ holds for all $x \in U$ and hence, $N_{C}^{\beta}(x)=N_{C}^{F}(x)=N_{C}^{L}(x)=N_{C}^{C l}(x)$ and $C$ is $\left(\|\cdot\|, N^{?}\right)$-hyporegular at $\bar{x}$ for any $N^{?}$ with $N^{?}(\cdot) \subset N^{C l}(\cdot)$.

Suppose that $\left(\|\cdot\|_{i}\right)_{i \in I}$ is a family of equivalent norms which are Fréchet differentiable outside zero and such that for some given $i_{0} \in I$, the norm $\|\cdot\|_{i_{0}}$ is uniformly smooth and uniformly convex. From the previous remark we know that if $C$ is $\left(\|\cdot\|_{i_{0}}, N^{P}\right)$-hyporegular at $\bar{x} \in C$ there exists some $\gamma>0$ and some open neighbourhood $U$ of $\bar{x}$ such that for all $x, x^{\prime} \in U \cap C$ and $x^{*} \in N_{i_{0}}^{L, s}(C ; x)$ with $\left\|x^{*}\right\|_{i_{0}, *} \leq 1$ (the dual norm of $\|\cdot\|_{i_{0}}$ )

$$
0 \geq\left\langle x^{*}, x^{\prime}-x\right\rangle-\gamma\left\|x^{\prime}-x_{0}\right\|_{i_{0}}^{2} .
$$


Then for the same open neighbourhood $U$ of $\bar{x}$, for each $i \in I$ there exists some $\gamma_{i}>0$ such that for all $x, x^{\prime} \in U \cap C$ and $x^{*} \in N^{F}(C ; x)$ with $\left\|x^{*}\right\|_{i, *} \leq 1$ one has

$$
0 \geq\left\langle x^{*}, x^{\prime}-x\right\rangle-\gamma_{i}\left\|x^{\prime}-x\right\|_{i}^{2} .
$$

Therefore for any $i \in I$ the set $C$ is $\left(\|\cdot\|_{i}, N^{P}\right)$-hyporegular at any $x \in U \cap C$ and for $\beta=2$ one has

$$
N_{i}^{P}(C ; x) \subset N_{C}^{F}(x)=N_{C}^{\beta}(x)=N_{C}^{C l}(x) \text { for all } x \in U \cap C .
$$

By specializing to certain families $\left(\|\cdot\|_{i}\right)_{i \in I}$ we have the next two results of the section. Here is the first one.

Proposition 5.3. Suppose that $\left(\|\cdot\|_{i}\right)_{i \in I}$ is a family of equivalent norms which are uniformly smooth and uniformly convex with the moduli of convexity $\delta_{\|\cdot\|_{i}}$ of power type $q=2$ for all $i \in I$.

If the closed set $C$ is $\left(\|\cdot\|_{i_{0}}, N^{P}\right)$-hyporegular at $\bar{x} \in C$ for some $i_{0} \in I$, then the following hold:

(a) the set $C$ is $\left(\|\cdot\|_{i}, N\right)$-hyporegular at $\bar{x}$ for any $i \in I$, and for any normal cone $N$ such that $N(\cdot) \subset N^{C l}(\cdot)$;

(b) the set $C$ is $\|\cdot\|_{i}$-prox-regular at $\bar{x}$ for any $i \in I$;

(c) there exists some neighbourhood $U$ of $\bar{x}$ such that for any $x \in U \cap C$ and any $i \in I$ and for $\beta=2$ the equalities $N_{C}^{\beta}(x)=N_{i}^{P}(C ; x)=N_{C}^{C l}(x)$ hold, and $C$ is $\left(\|\cdot\|_{i}, N\right)$-hyporegular at $x$ for any normal cone $N_{C}(\cdot)$ with $N_{C}^{\beta}(\cdot) \subset N_{C}(\cdot) \subset N_{C}^{C l}(\cdot)$.

Proof. The first point has been seen above. The second and third results come from the first one, from (5.4), and from Corollary 5.1.

We specialize further, to the case of $\mathbb{R}^{n}$ endowed with the family $\left(\|\cdot\|_{p}\right)_{p>1}$ of classical $l_{p}$-norms, say $\|x\|_{p}=\left(\sum_{k=1}^{p}\left|x_{k}\right|^{p}\right)^{1 / p}$.

Proposition 5.4. Suppose that $X=\mathbb{R}^{n}$ and that $\left(\|\cdot\|_{p}\right)_{p>1}$ is the family of $l_{p^{-}}$ norms with $p>1$.

1) If for some $p_{0}>1$ the set $C$ is $\left(\|\cdot\|_{p_{0}}, N^{P}\right)$-hyporegular at $\bar{x} \in C$, then (a) the set $C$ is $\left(\|\cdot\|_{p}, N\right)$-hyporegular at $\bar{x}$ for any $p>1$ and any normal cone $N$ such that $N(\cdot) \subset N^{C l}(\cdot)$;

(b) the set $C$ is $\|\cdot\|_{p}$-prox-regular at $\bar{x}$ for any $\left.\left.p \in\right] 1,2\right]$;

(c) there is some neighbourhood $U$ of $\bar{x}$ such that for all $p \in] 1,2]$ and $p^{\prime}>2$ and for $\beta=2$ one has

$$
N_{p^{\prime}}^{P}(C ; x) \subset N_{p}^{P}(C ; x)=N_{C}^{\beta}(x)=N_{C}^{C l}(x) \quad \text { for any } x \in U .
$$

2) If for some $p_{0} \geq 2$ the set $C$ is $\|\cdot\|_{p_{0}}$-prox-regular at $\bar{x} \in C$, then $\left(a^{\prime}\right)$ the set $C$ is $\left(\|\cdot\|_{p}, N\right)$-hyporegular at $\bar{x}$ for any $p>1$ and any normal cone $N$ such that $N(\cdot) \subset N^{C l}(\cdot)$;

$\left(b^{\prime}\right)$ the set $C$ is $\|\cdot\|_{p}$-prox-regular at $\bar{x}$ for any $\left.\left.p \in\right] 1,2\right]$;

$\left(c^{\prime}\right)$ there is some neighbourhood $U$ of $\bar{x}$ such that for all $\left.\left.p \in\right] 1,2\right]$ and $p^{\prime}>2$ and for $\beta=2$ one has

$$
N_{p^{\prime}}^{P}(C ; x) \subset N_{p}^{P}(C ; x)=N_{p_{0}}^{P}(C ; x)=N_{C}^{2} \beta(x)=N_{C}^{C l}(x) \quad \text { for any } x \in U .
$$

Proof. Under the assumption of the first case, the property (a) like (a) in Proposition 5.3 has been seen earlier. The second property (b) is a consequence of (b) in Proposition 5.3 since for any $p \in] 1,2]$, the norm $\|\cdot\|_{p}$ has modulus of convexity of 
power type $q=2$. Taking the open neighbourhood $U$ given by (c) of Proposition 5.3 for the family of norms $\left(\|\cdot\|_{p}\right)_{p \in] 1,2]}$, only the first inclusion of the third property

(c) remains to be argued. It is a consequence of $C$ being $\left(\|\cdot\|_{p^{\prime}}, N^{P}\right)$-hyporegular at any $x \in U \cap C$ for any $p^{\prime}>2$ according to the property (a), which entails for $\beta=2$ the inclusion $N_{p^{\prime}}^{P}(C ; x) \subset N_{C}^{\beta}(x)$ for $\beta=2$ and for any $x \in U \cap C$.

Now concerning the second case, suppose that for some $p_{0} \geq 2$ the set $C$ is $\|\cdot\|_{p_{0}}$ prox-regular at $\bar{x} \in C$. We first observe that Definition 2.1 furnishes some open neighbourhood $U$ of $\bar{x}$ such that $C$ is $\|\cdot\|_{p_{0}}$-prox-regular at any point $x \in C \cap U$. Since the norm $\|\cdot\|_{p_{0}}$ is uniformly smooth with modulus of smoothness $s=2$, Proposition 5.2 yields that $C$ is $N^{F}$-hyporegular at any $x \in C \cap U$ relative to the family $\left(\|\cdot\|_{p}\right)_{p>1}$ and that $N_{p_{0}}^{P}(C ; x)=N_{C}^{F}(x)$ for any $x \in U$. Then for any $p>1$ the set $C$ is $\left(\|\cdot\|_{p}, N^{P}\right)$-hyporegular at any $x \in C \cap U$ and then from the first case and what precedes, we have the second and third properties $\left(\mathrm{b}^{\prime}\right)$ and $\left(\mathrm{c}^{\prime}\right)$.

\section{Comparison of normal CONES}

The proposition of this section compares, for any closed subset $C$ of $X$, the cone $N_{C}^{P}(\cdot)$ of proximal normal functionals with the normal cone $N_{C}^{\beta}(\cdot)$ when $\beta$ is the power type of the modulus of uniform convexity or of smoothness of the norm $\|\cdot\|$ of $X$.

Proposition 6.1. Let $C$ be a closed subset of $X$ and let $x \in C$.

(a) If the modulus of convexity of the norm $\|\cdot\|$ of $X$ is of power type $q$, then

$$
N_{C}^{q}(x) \subset N_{C}^{P}(x) .
$$

(b) Similarly, if the modulus of smoothness of the norm $\|\cdot\|$ of $X$ is of power type $s$, then

$$
N_{C}^{P}(x) \subset N_{C}^{s}(x) .
$$

Proof. Suppose that the modulus of convexity of $\|\cdot\|$ is of power type $q$. From (2.4), there exists some constant $K>0$ such that for all $x, y \in X$,

$$
\|x+y\|^{q} \geq\|x\|^{q}+q\left\langle J_{q}(x), y\right\rangle+K\|y\|^{q} .
$$

Take $x^{*} \in N_{C}^{q}(x), x^{*} \neq 0$. There exist $\gamma, \varepsilon>0$ such that for any $x^{\prime} \in B(x, \varepsilon) \cap C$, $\left\langle x^{*}, x^{\prime}-x\right\rangle \leq \gamma\left\|x^{\prime}-x\right\|^{q}$. For any $x^{\prime} \in B(x, \varepsilon) \cap C$ we then have for each $t>0$,

$$
\left\|x+t J_{q}^{-1}\left(x^{*}\right)-x^{\prime}\right\|^{q} \geq\left\|t J_{q}^{-1}\left(x^{*}\right)\right\|^{q}+q\left\langle J_{q}\left(t J_{q}^{-1}\left(x^{*}\right)\right), x-x^{\prime}\right\rangle+K\left\|x-x^{\prime}\right\|^{q} ;
$$

that is, by the equality in (2.1),

$$
\left\|x+t J_{q}^{-1}\left(x^{*}\right)-x^{\prime}\right\|^{q} \geq\left\|t J_{q}^{-1}\left(x^{*}\right)\right\|^{q}+t^{q-1} q\left\langle J_{q}\left(J_{q}^{-1}\left(x^{*}\right)\right), x-x^{\prime}\right\rangle+K\left\|x-x^{\prime}\right\|^{q} .
$$

Hence

$$
\left\|x+t J_{q}^{-1}\left(x^{*}\right)-x^{\prime}\right\|^{q} \geq\left\|t J_{q}^{-1}\left(x^{*}\right)\right\|^{q}-t^{q-1} q \gamma\left\|x^{\prime}-x\right\|^{q}+K\left\|x-x^{\prime}\right\|^{q},
$$

so, whenever $t \leq(K /(\rho \gamma))^{1 /(q-1)}$, we obtain

$$
\left\|x+t J_{q}^{-1}\left(x^{*}\right)-x^{\prime}\right\|^{q} \geq\left\|t J_{q}^{-1}\left(x^{*}\right)\right\|^{q} .
$$

Because of the local character (2.11) of the primal normal vector, the latter equality entails that $J_{q}^{-1}\left(x^{*}\right) \in P N_{C}(x)$, that is, $J\left(J_{q}^{-1}\left(x^{*}\right)\right) \in N_{C}^{P}(x)$. As $J\left(J_{q}^{-1}\left(x^{*}\right)\right) \in$ $\mathbb{R}_{+} x^{*}$, the first inclusion follows. 
Now to prove (b) fix any $x^{*} \in N_{C}^{P}(x)$ with $x^{*} \neq 0$. By definition of $N_{C}^{P}(\cdot)$, there exists $\delta>0$ such that for any $t \in] 0, \delta]$ and any $x^{\prime} \in C$,

$$
\left\|x+t J^{-1}\left(x^{*}\right)-x^{\prime}\right\|^{s} \geq\left\|t J^{-1}\left(x^{*}\right)\right\|^{s} .
$$

From (2.5), there exists some constant $L>0$ such that, for every $x, y \in X$,

$$
\|x+y\|^{s} \leq\|x\|^{s}+s\left\langle J_{s}(x), y\right\rangle+L\|y\|^{s} .
$$

From the two previous estimations we derive for any $x^{\prime} \in C$,

$$
s\left\langle J_{s}\left(\delta J^{-1}\left(x^{*}\right)\right), x-x^{\prime}\right\rangle+L\left\|x-x^{\prime}\right\|^{s} \geq 0 .
$$

That is, according to the equality in (2.1),

$$
s \delta^{s-1}\left\langle J_{s}\left(J^{-1}\left(x^{*}\right)\right), x-x^{\prime}\right\rangle+L\left\|x-x^{\prime}\right\|^{s} \geq 0 .
$$

As $J_{s}\left(J^{-1}\left(x^{*}\right)\right) \in \mathbb{R}_{+} x^{*}$, we deduce that there exists some $\sigma>0$ such that

$$
\left\langle x^{*}, x^{\prime}-x\right\rangle \leq \sigma\left\|x^{\prime}-x\right\|^{s} \quad \text { for all } x^{\prime} \in C .
$$

This entails that $x^{*} \in N_{C}^{s}(x)$ and the inclusion of (b) is proved.

\section{Preservation of hyporegularity and PROX-REgUlarity}

This section is devoted to the study of the preservation of prox-regularity for the intersection of finitely many sets and for the inverse image. The characterizations of prox-regularity in Theorem 2.3 with the property (ii) of hypomonotonicity of the proximal normal cone as well as the property (iii) of local single-valuedness and continuity of the metric projection mapping have been crucial in the study 24. of differential inclusions of sweeping process type governed by nonconvex proxregular sets. The regularization of such differential inclusions in Hilbert space in 43] uses the property $(i v)$ of the same theorem. Below we will take advantage of the property $(i)$ to investigate the stability of metrical prox-regularity under the above set-operations. In fact we will start with the stability of $N^{P}$-hyporegularity through the property (5.1) which is in line with property $(i)$ of Theorem 2.3 ,

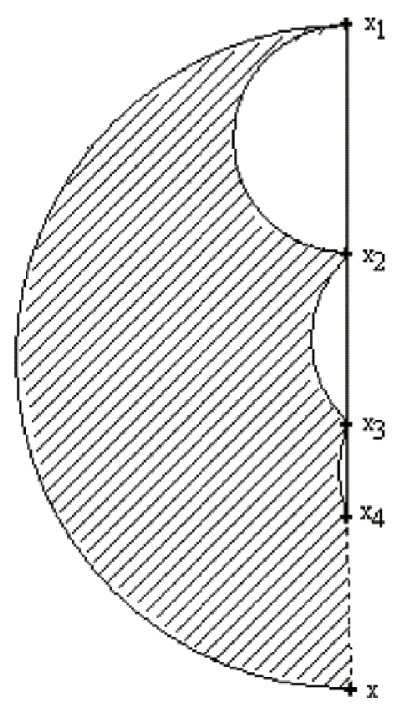

FiguRE 1. Intersection of prox-regular sets 
To see first that the intersection of finitely many prox-regular sets may not be prox-regular, consider the example in $\mathbb{R}^{2}$ with its Euclidean norm illustrated by Figure 1, where the set $C_{1}$ is the whole line $\left(x_{1} x_{2}\right),\left(x_{i}\right)_{i}$ being a sequence of points on $C_{1}$ that converges to some $x \in C_{1}$, and the set $C_{2}$ is the hatched surface delimited on one side by the closed arc $\overparen{x_{1} x}$ of the half circle with radius $R=\left\|x_{1}-x\right\| / 2$ and on its other side by the arcs of the circles (all with the same radius $r<R$ )

$x_{i} \widehat{x_{i+1}}, i \in \mathbb{N}$, so that $x$ belongs also to $C_{2}$. It is easily seen that while $C_{1}$ and $C_{2}$ are both prox-regular at $x$ (they are even uniformly prox-regular), their intersection $C_{1} \cap C_{2}$ is not prox-regular at the point $x$.

To provide general sufficient conditions under which the prox-regularity of intersection or inverse image is preserved, we need first to recall the concept of calmness. Translating the concept of calmness of a multivalued mapping in 40] we say that the intersection of a finite family of sets $\left(C_{k}\right)_{k=1}^{m}$ is metrically calm at a point $\bar{x} \in \bigcap_{k=1}^{m} C_{k}$ provided there exist a constant $\gamma>0$ and a neighbourhood $U$ of $\bar{x}$ such that

$$
d\left(x, \bigcap_{k=1}^{m} C_{k}\right) \leq \gamma\left(d\left(x, C_{1}\right)+\cdots+d\left(x, C_{m}\right)\right) \quad \text { for all } x \in U .
$$

Now let $F: X \rightarrow Y$ be a mapping from $X$ into another uniformly convex Banach space $Y$ and let $D$ be a subset of $Y$ and $\bar{x} \in F^{-1}(D)$. As above we say that the mapping $F$ is metrically calm at $\bar{x}$ relative to the set $D$ when there exist a constant $\gamma>0$ and a neighbourhood $U$ of $\bar{x}$ such that

$$
d\left(x, F^{-1}(D)\right) \leq \gamma d(F(x), D) \text { for all } x \in U .
$$

Proposition 7.1. Let $\left(C_{k}\right)_{k=1}^{m}$ be a finite family of closed sets of $X$ and let $D$ be a closed set of $Y$.

(a) If all sets $C_{k}$ are $N^{P}$-hyporegular at a point $\bar{x}$ of all the sets $C_{k}$ and if the intersection is metrically calm at $\bar{x}$, then this intersection set $\bigcap_{k=1}^{m} C_{k}$ is $N^{P}$ hyporegular at $\bar{x}$ too.

(b) If a mapping $F: X \rightarrow Y$ is of class $C^{1,1}$ around a point $\bar{x} \in F^{-1}(D)$ and metrically calm at $\bar{x}$ relative to $D$ and if $D$ is $N^{P}$-hyporegular at $F(\bar{x})$, then the set $F^{-1}(D)$ is $N^{P}$-hyporegular at $\bar{x}$.

Proof. Assume that each set $C_{k}$ is $N^{P}$-hyporegular at $\bar{x}$ and put $C:=\bigcap_{k=1}^{m} C_{k}$. By definition of $N^{P}$-hyporegularity (see (5.1) ) it is easily seen that there exist some real number $r>0$ and some open neighbourhood $U$ of $\bar{x}$ such that for each $k=1, \ldots, m$ we have for all $x \in C_{k} \cap U$ and $u^{*} \in N_{C_{k}}^{P}(x) \cap \mathbb{B}^{*}$,

$$
\left\langle u^{*}, x^{\prime}-x\right\rangle \leq(2 r)^{-1}\left\|x^{\prime}-x\right\|^{2} \quad \text { for all } x^{\prime} \in C_{k} \cap U .
$$

Restricting the neighbourhood $U$ if necessary, we may suppose that the inequality (17.1) holds upon $U$. Fix any $x \in C \cap U$ and take any $x^{*} \in N_{C}^{P}(x)$ with $\left\|x^{*}\right\| \leq$ 1. We have that $x^{*} \in N_{C}^{F}(x)$ and hence $x^{*} \in \partial_{F} d_{C}(x)$ since one knows that $\partial_{F} d_{C}(x)=N_{C}^{F}(x) \cap \mathbb{B}^{*}$ (see, e.g., [35, Corollary 1.96]). This inequality (7.1) and the definition of Fréchet subgradient easily yield that $x^{*} \in \gamma \partial_{F}\left(d_{C_{1}}+\cdots+d_{C_{m}}\right)(x)$ and hence in particular $x^{*} \in \gamma \partial_{L}\left(d_{C_{1}}+\cdots+d_{C_{m}}\right)(x)$. The functions $d_{C_{k}}$ being Lipschitzian, the formula of the Mordukhovich subdifferential of a finite sum of locally Lipschitzian functions (see, e.g., [35, Theorem 3.36]) ensures us that there exist $u_{k}^{*} \in \partial_{L} d_{C_{k}}(x)$ such that $\gamma^{-1} x^{*}=u_{1}^{*}+\cdots+u_{m}^{*}$. Restricting again the neighbouhood $U$, by Theorem 3.2 we have $u_{k}^{*} \in \partial_{P} d_{C_{k}}(x)=N_{C}^{P}(x) \cap \mathbb{B}^{*}$, which by 
(17.3) gives $\left\langle u_{k}^{*}, x^{\prime}-x\right\rangle \leq(2 r)^{-1}\left\|x^{\prime}-x\right\|^{2}$ for every $x^{\prime} \in C_{k} \cap U$. Consequently for any $x^{\prime} \in C \cap U$ we obtain

$$
\left\langle\gamma^{-1} x^{*}, x^{\prime}-x\right\rangle \leq m(2 r)^{-1}\left\|x^{\prime}-x\right\|^{2},
$$

and this obviously implies that the intersection set $C$ is $N^{P}$-hyporegular at $\bar{x}$; that is, (a) is proven.

Let us now establish (b). Fix some open convex neighbourhood $U$ of $\bar{x}$ over which (7.2) holds and over which the mapping $F$ as well as its derivative $D F(\cdot)$ are Lipschitzian with Lipschitzian constants $K$ and $K_{1}$ respectively. For $S:=$ $F^{-1}(D)$, fix any $x \in U \cap S$ and $x^{*} \in N_{S}^{P}(x)$ with $\left\|x^{*}\right\| \leq 1$. As above we have $x^{*} \in \partial_{F} d_{S}(x)$ and this entails by (7.2) that $x^{*} \in \gamma \partial_{F}\left(d_{D} \circ F\right)(x)$ and hence $x^{*} \in$ $\gamma \partial_{L}\left(d_{D} \circ F\right)(x)$. The subdifferential chain rule (see, e.g., [35. Corollary 3.43]) gives some $y^{*} \in \partial_{L} d_{D}(F(x))$ such that $\gamma^{-1} x^{*}=y^{*} \circ D F(x)$. Fix by (5.1) some open neighbourhood $V$ of $F(\bar{x})$ and some constant $r>0$ such that

$\left\langle v^{*}, y^{\prime}-y\right\rangle \leq(2 r)^{-1}\left\|y^{\prime}-y\right\|^{2} \quad$ for all $y \in V \cap D, y^{\prime} \in V \cap D$ and $v^{*} \in N_{D}^{P}(y) \cap \mathbb{B}^{*}$, and such that the equality concerning the subdifferentials of the distance function in Theorem 3.2 holds with $d_{D}$ at all points of $V \cap D$. Restricting the open convex neighbourhood $U$ of $\bar{x}$ if necessary, we may suppose that $F(U) \subset V$ and that $\partial_{L} d_{D}(F(u))=\partial_{P} d_{D}(F(u))$ for all $u \in U \cap S$ according to Theorem 3.2. Then (7.4) yields

$$
\left\langle y^{*}, y^{\prime}-F(x)\right\rangle \leq(2 r)^{-1}\left\|y^{\prime}-F(x)\right\|^{2} \quad \text { for all } y^{\prime} \in V \cap D .
$$

Fix any $x^{\prime} \in S \cap U$ and write

$$
F\left(x^{\prime}\right)-F(x)=D F(x)\left(x^{\prime}-x\right)+\int_{0}^{1}\left(D F\left(x+t\left(x^{\prime}-x\right)\right)-D F(x)\right)\left(x^{\prime}-x\right) d t
$$

and then

$\left\langle\gamma^{-1} x^{*}, x^{\prime}-x\right\rangle=\left\langle y^{*}, F\left(x^{\prime}\right)-F(x)\right\rangle-\int_{0}^{1}\left\langle y^{*} \circ\left(D F\left(x+t\left(x^{\prime}-x\right)\right)-D F(x)\right), x^{\prime}-x\right\rangle d t$.

Using (7.5) we obtain

$\left\langle x^{*}, x^{\prime}-x\right\rangle \leq \gamma(2 r)^{-1}\left\|F\left(x^{\prime}\right)-F(x)\right\|^{2}+\gamma K_{1}\left\|x^{\prime}-x\right\|^{2} \leq \gamma\left(K^{2}(2 r)^{-1}+K_{1}\right)\left\|x^{\prime}-x\right\|^{2}$.

The latter being true for all $x, x^{\prime} \in S \cap U$ and $x^{*} \in N_{S}^{P}(x) \cap \mathbb{B}^{*}$, we conclude that the set $S$ is $N^{P}$-hyporegular at $\bar{x}$.

For the convenience of the reader we made the choice to prove first the $N^{P}$ hyporegularity of the intersection and then to give the additional arguments yielding the $N^{P}$-hyporegularity of the inverse image. However the case of the intersection can be derived from that of the inverse image. Indeed putting $Y=X^{m}, F(x)=$ $(x, \ldots, x)$ for all $x \in X$, and $D=C_{1} \times \cdots \times C_{m}$ we see that $F^{-1}(D)=\bigcap_{k=1}^{m} C_{k}$ and then some direct arguments and computation allow us to obtain through $(b)$ the result of $(a)$. 5.1.

We now state the following corollary which is a direct consequence of Corollary

Corollary 7.2. Assume that the moduli of convexity and smoothness of the norm of $X$ are of power type and the power type of convexity is $q=2$. Then under the assumptions of Proposition [7.1, the sets $\bigcap_{k=1}^{m} C_{k}$ and $F^{-1}(D)$ are prox-regular at $\bar{x}$. 
When the spaces $(X,\|\cdot\|)$ and $(Y,\|\cdot\|)$ are Hilbert spaces, Proposition 7.1 translates, according to Corollary 5.1 and Proposition 5.2, the preservation of proxregularity.

Corollary 7.3. Assume that $X$ and $Y$ are Hilbert spaces for their respective norms. Let $\left(C_{k}\right)_{k=1}^{m}$ be a finite family of closed sets of $X$ and let $D$ be a closed set of $Y$.

(a) If all sets $C_{k}$ are prox-regular at a point $\bar{x}$ of all the sets $C_{k}$ and if the intersection is metrically calm at $\bar{x}$, then this intersection set $\bigcap_{k=1}^{m} C_{k}$ is proxregular at the point $\bar{x}$.

(b) If a mapping $F: X \rightarrow Y$ is of class $C^{1,1}$ around a point $\bar{x} \in F^{-1}(D)$ and metrically calm at $\bar{x}$ relative to $D$ and if $D$ is prox-regular at $F(\bar{x})$, then the set $F^{-1}(D)$ is prox-regular at $\bar{x}$.

An analysis of the proof of Proposition 7.1 reveals that a uniform version also holds. We state it only in the case of an intersection and leave the case of the inverse image to the reader.

Proposition 7.4. Assume that all the closed sets $C_{k}, k=1, \ldots, m$, are $r$-uniformly $N^{P}$-hyporegular (resp. $r$-uniformly prox-regular and $(X,\|\cdot\|)$ is a Hilbert space) and that their intersection is calm at any one of its points with the same modulus $\gamma$ of metric calmness in (7.1). Then the set $\bigcap_{k=1}^{m} C_{k}$ is $r^{\prime}$-uniformly $N^{P}$-hyporegular (resp. $r^{\prime}$-uniformly metrically prox-regular) with $r^{\prime}:=r /(m \gamma)$.

In the literature there are conditions with normal cones easy to handle ensuring the metric calmness inequalities (7.1) and (7.2). For example, such a condition for (7.1) is known (see, e.g., [28, pp. 548-549]) under the name of general position condition for intersection of finitely many sets. In the context of our uniformly convex Banach space, the concept can be translated by saying that the closed sets $C_{1}, \ldots, C_{m}$ are (relative to the Fréchet normal cone) in sequential general position at $\bar{x} \in \bigcap_{k=1}^{m} C_{k}$ whenever for any sequence of tuples $\left(x_{1, n}, \ldots, x_{m, n}, x_{1, n}^{*}, \ldots, x_{m, n}^{*}\right)_{n}$ such that $x_{k, n} \in C_{k}, x_{k, n} \rightarrow \bar{x}, x_{k, n}^{*} \in N_{C_{k}}^{F}\left(x_{k, n}\right) \cap \mathbb{B}^{*}$ and such that $\left\|\sum_{k=1}^{m} x_{k, n}^{*}\right\| \rightarrow 0$, one has $\left\|x_{k, n}^{*}\right\| \rightarrow 0$ for any $k=1, \ldots, m$.

Another condition which is much easier to handle involves as above a property with normal cones but at the fixed point $\bar{x}$. It probably appears for the first time as one of the assumptions of Theorem 4.10 of Federer's seminal paper [26] for sets of $\mathbb{R}^{n}$ which are not submanifolds. The sets $C_{k}, k=1, \ldots, m$, are (relative to the limiting normal cone) in pointbased general position at $\bar{x}$ provided that the equality $x_{1}^{*}+\cdots+x_{m}^{*}=0$ with $x_{k}^{*} \in N_{C_{k}}^{L}\left(x_{k}\right)$ entails $x_{1}^{*}=\cdots=x_{m}^{*}=0$.

Now recall that a closed set of $X$ is compactly epi-Lipschitzian at $\bar{x} \in C$ (a concept due to J. M. Borwein and H. M. Strójwas [11]) if there exist a compact set $Q$, a neighbourhood $V$ of zero, a neighbourhood $U$ of $\bar{x}$ in $X$, and a positive real number $\varepsilon$ such that

$$
C \cap U+t V \subset C+t Q \text { for all } t \in] 0, \varepsilon] .
$$

Of course any closed subset of $X$ is compactly epi-Lipschitzian at any of its points whenever the space $X$ is finite dimensional.

Corollary 7.5. Let $\left(C_{k}\right)_{k=1}^{m}$ be a finite family of closed sets of $X$ and let $\bar{x} \in$ $\bigcap_{k=1}^{m} C_{k}$. Then the following hold. 
(a) If the sets $C_{k}$ are in sequential general position at $\bar{x}$ and if each set $C_{k}$ is $N^{P}$-hyporegular at $\bar{x}$ (resp. prox-regular at $\bar{x}$ and $(X,\|\cdot\|)$ is a Hilbert space), then the intersection $\bigcap_{k=1}^{m} C_{k}$ is $N^{p}$-hyporegular (resp. prox-regular) at $\bar{x}$.

(b) If all the sets $C_{k}$ except at most one are compactly epi-Lipschitzian at $\bar{x}$, then the sequential general position in (a) may be replaced by the corresponding pointbased general position at $\bar{x}$.

(c) If the space $X$ is finite dimensional, again the sequential general position in (a) may be replaced by the corresponding pointbased general position at $\bar{x}$.

Proof. (a) Since the uniformly convex Banach space $X$ is an Asplund space, the sequential general position is known to entail the metric calmness property (7.1) (and even more) according to Proposition 6 on p. 548 and Theorem 2 on p. 545 of 27. (for example). Then the conclusion of $(a)$ follows from Proposition 7.1 (resp. Corollary 7.3 .

(b) The result comes from the fact that the assumption of the compactly epi-Lipschitzian property combined with the pointbased general position at $\bar{x}$ ensures (see, e.g., [30, Theorem 3.4]) that the intersection is metrically regular and hence metrically calm at $\bar{x}$.

(c) This is a direct consequence of $(b)$.

The result in (c) was previously established in (5) of Theorem 4.10 in 26 .

\section{Conical Derivative of the mapping $P_{C}$}

The following result is well known for convex sets of Hilbert space (see Zarantonello [46, p. 300]). The term conical derivative has been coined on page 301 of [46. The result has been independently extended by Canino [15] to closed $p$-convex sets of Hilbert space and by Shapiro [41] to closed sets with the 2-order tangential property of Hilbert space too. It is actually known (see [39]) that, in the context of Hilbert space, the concept of $p$-convex set is equivalent to uniform prox-regularity and the 2-order tangential property is equivalent to the local prox-regularity. The related result in 41 may then be translated for prox-regular sets. Note also that the proof in [15. still holds for sets which are prox-regular at the considered point of the Hilbert space. Our proposition below deals with the context of uniformly convex space.

Proposition 8.1. Assume that the moduli of uniform convexity and smoothness of the norm $\|\cdot\|$ of $X$ are of power type. Assume also that the closed set $C$ of $X$ is prox-regular at $\bar{x} \in C$. Then there exists an open neighbourhood $U$ of $\bar{x}$ such that $P_{C}$ is single-valued and continuous on $U$ and such that for any $x \in U \cap C$ and $y \in X$ for which the mapping $t \mapsto \frac{1}{t}\left[P_{C}(x+t y)-x\right]$ is bounded on some interval ] $\left.0, t_{0}\right]$, one has

$$
\lim _{t \downarrow 0} \frac{1}{t}\left[P_{C}(x+t y)-x\right]=P_{K_{C}(x)}(y)
$$

and the directional derivative $d_{C}^{\prime}(x ; y):=\lim _{t \downarrow 0} \frac{1}{t}\left[d_{C}(x+t y)-d_{C}(x)\right]$ of $d_{C}$ at $x$ in the direction $y$ exists and

$$
d_{C}^{\prime}(x ; y)=d\left(y, K_{C}(x)\right),
$$

which translates in the terminology of [46] that $d_{C}^{\prime}(x ; \cdot)$ is a conical derivative. 
Proof. By the inequality (3.12) of the proof of Theorem 3.2, there exist $\varepsilon, r>0$ with $\varepsilon<1 / 2$ such that $P_{C}$ is a single-valued continuous mapping on $B(\bar{x}, \varepsilon)$ and

$$
\left\langle x^{*}-u^{*}, u-x\right\rangle \leq \frac{2}{r}\|x-u\| \omega_{r+1}(\|x-u\|)
$$

for all $x, u \in B(\bar{x}, \varepsilon)$ and all $x^{*} \in N_{C}^{P}(x), u^{*} \in N_{C}^{P}(u)$ with $\left\|x^{*}\right\| \leq 1$ and $\left\|u^{*}\right\| \leq 1$, where $\omega_{r+1}$ is the modulus of uniform continuity of the duality mapping $J$ over the bounded set $(r+1) \mathbb{B}$. Let $x \in B(\bar{x}, \varepsilon) \cap C, y \in X$, and $t_{0}>0$ such that the mapping $t \mapsto \frac{1}{t}\left[P_{C}(x+t y)-x\right]$ is bounded on $\left.] 0, t_{0}\right]$, say by $\beta>0$. We may suppose that for all $\left.t \in] 0, t_{0}\right]$ we have $x+t y \in B(\bar{x}, \varepsilon)$ and $P_{C}(x+t y) \in B(\bar{x}, \varepsilon)$. For any $\left.\left.t \in\right] 0, t_{0}\right]$, putting $h_{t}^{\prime}:=\frac{1}{t}\left[P_{C}(x+t y)-x\right]$, we see that

$$
y-h_{t}^{\prime}=\frac{1}{t}\left[x+t y-P_{C}(x+t y)\right]
$$

and hence $y-h_{t}^{\prime} \in P N_{C}\left(P_{C}(x+t y)\right)$, i.e., $J\left(y-h_{t}^{\prime}\right) \in N_{C}^{P}\left(P_{C}(x+t y)\right)$.

Now take any sequence $\left(t_{n}\right)_{n}$ of $\left.] 0, t_{0}\right]$ converging to 0 . The sequence $\left(J\left(y-h_{t_{n}}^{\prime}\right)\right)_{n}$ being bounded, there exists an increasing function $\sigma: \mathbb{N} \rightarrow \mathbb{N}$ such that the sequence $\left(J\left(y-h_{t_{\sigma(n)}}^{\prime}\right)\right)_{n}$ converges weakly star to $J(y-h)$ for some vector $h \in X$. As $N_{C}^{P}(x)=N_{C}^{L}(x)$ (see Theorem 3.2 ), we have $J(y-h) \in N_{C}^{P}(x)$. Put $h_{n}:=h_{t_{\sigma(n)}^{\prime}}^{\prime}$. By (8.1) there exists some constant $\gamma>0$ independent of $n$ such that for $\lambda_{n}:=t_{\sigma(n)}$, $\left\langle J\left(y-h_{n}\right)-J(y-h), x-P_{C}\left(x+\lambda_{n} y\right)\right\rangle \leq \frac{\gamma}{r}\left\|P_{C}\left(x+\lambda_{n} y\right)-x\right\| \omega_{r+1}\left(\left\|P_{C}\left(x+\lambda_{n} y\right)-x\right\|\right)$.

The latter inequality is equivalent to

$$
\left\langle J\left(y-h_{n}\right)-J(y-h),-\lambda_{n} h_{n}\right\rangle \leq \frac{\gamma}{r}\left\|P_{C}\left(x+\lambda_{n} y\right)-x\right\| \omega_{r+1}\left(\left\|P_{C}\left(x+\lambda_{n} y\right)-x\right\|\right),
$$

that is,

$$
\begin{aligned}
& \left\langle J\left(y-h_{n}\right)-J(y-h),\left(y-h_{n}\right)-(y-h)\right\rangle-\left\langle J\left(y-h_{n}\right)-J(y-h), h\right\rangle \\
& \leq \frac{\gamma}{r}\left\|\frac{1}{\lambda_{n}}\left[P_{C}\left(x+\lambda_{n} y\right)-x\right]\right\| \omega_{r+1}\left(\left\|P_{C}\left(x+\lambda_{n} y\right)-x\right\|\right) .
\end{aligned}
$$

If $\max \left\{\left\|y-h_{n}\right\|,\|y-h\|\right\}=0$ for infinitely many $n$, then for all these integers $n$ we have $y=h_{n}=h$ and hence by definition of $h_{n}$ we obtain $h=\frac{1}{\lambda_{n}}\left[P_{C}\left(x+\lambda_{n} y\right)-x\right]$, which yields $h \in K_{C}(x)$. Suppose now that $\max \left\{\left\|y-h_{n}\right\|,\|y-h\|\right\}>0$ for all $n$ not less than some $n_{0}$. According to (2.2) for some constant $K_{2}>0$ we have for all $n \geq n_{0}$,

$$
\begin{aligned}
& K_{2}\left(\max \left\{\left\|y-h_{n}\right\|,\|y-h\|\right\}\right)^{2} \delta_{\|\cdot\|}\left(\frac{\left\|h_{n}-h\right\|}{2\left(\max \left\{\left\|y-h_{n}\right\|,\|y-h\|\right\}\right)}\right) \\
& \leq\left\langle J\left(y-h_{n}\right)-J(y-h), h\right\rangle+\frac{\gamma}{r}\left\|\frac{1}{\lambda_{n}}\left[P_{C}\left(x+\lambda_{n} y\right)-x\right]\right\| \omega_{r+1}\left(\left\|P_{C}\left(x+\lambda_{n} y\right)-x\right\|\right),
\end{aligned}
$$

and since the first expression of the second member tends to 0 and the modulus of convexity $\delta_{\|\cdot\|}$ is an increasing function with $\delta_{\|\cdot\|}(t) \underset{t \downarrow 0}{\longrightarrow} 0$, it is not difficult to see that $\left\|h_{n}-h\right\| \rightarrow 0$ and by definition of $h_{n}$ we obtain $h \in K_{C}(x)$. So in any case, we have $h \in K_{C}(x)$ and the sequence $\left(h_{n}\right)_{n}$ strongly converges to $h$.

Now take any $v \in K_{C}(x)$. Since $K_{C}(x)=T_{C}(x)$ according to Theorem 3.2 . there is a sequence $\left(z_{n}\right)_{n}$ converging to $v$ such that $x+\lambda_{n} z_{n} \in C$ for all $n$. This entails that for all $n$,

$$
\left\|x+\lambda_{n} y-\left(x+\lambda_{n} z_{n}\right)\right\| \geq\left\|x+\lambda_{n} y-P_{C}\left(x+\lambda_{n} y\right)\right\|,
$$


which is equivalent to

$$
\left\|y-z_{n}\right\| \geq\left\|y+\frac{1}{\lambda_{n}}\left[x-P_{C}\left(x+\lambda_{n} y\right)\right]\right\| .
$$

Then passing to the limit with $n \rightarrow \infty$, we obtain $\|y-v\| \geq\|y-h\|$ for all $v \in K_{C}(x)$, which means that $h=P_{K_{C}(x)}(y)$. So for any sequence of positive numbers $\left(t_{n}\right)_{n}$ converging to 0 there exists a subsequence $\left(t_{\sigma(n)}\right)_{n}$ such that

$$
\lim _{n \rightarrow \infty} \frac{1}{t_{\sigma(n)}}\left[P_{C}\left(x+t_{\sigma(n)} y\right)-x\right]=P_{K_{C}(x)}(y) .
$$

Consequently

$$
\lim _{t \downarrow 0} \frac{1}{t}\left[P_{C}(x+t y)-x\right]=P_{K_{C}(x)}(y) .
$$

Concerning the equality of the directional derivative of the distance function to the set $C$, observe first that for any positive number $t$ small enough,

$$
\begin{aligned}
\frac{1}{t}\left[d_{C}(x+t y)-d_{C}(x)\right]=\frac{1}{t} d_{C}(x+t y) & =\frac{1}{t}\left\|P_{C}(x+t y)-(x+t y)\right\| \\
& =\left\|\frac{1}{t}\left[P_{C}(x+t y)-x\right]-y\right\| .
\end{aligned}
$$

Then taking (8.2) into account and passing to the limit when $t \downarrow 0$ give that the directional derivative $d_{C}^{\prime}(x ; y$ exists and

$$
d_{C}^{\prime}(x ; y)=\left\|P_{K_{C}(x)}(y)-y\right\|=d\left(y, K_{C}(x)\right) .
$$

In the case of a Hilbert space $X$ the mapping $P_{C}$ is Lipschitzian near $\bar{x}$ (see 39] ) and hence the boundedness assumption of the proposition above is fulfilled and one recovers in Corollary 8.2 below the result of Proposition 2.12 in [15] and Theorem 3.1 in 41]. The result is proved in 41] through some properties of solutions of perturbed optimization problems satisfying some appropriate conditions. The proof in [15] is based on the duality in Hilbert space between the tangent cone $K_{C}(x)$ and the primal normal cone $P N_{C}(x)$ for prox-regular sets $C$. Such a duality property is not available in the non-Hilbert setting because of the nonlinearity of the duality mapping $J$. Instead, our proof of Proposition 8.1 above is related to the tangential regularity of $C$ established in Theorem 3.2 and to the sequential characterization of the Clarke tangent cone (recalled at the beginning of Section 3).

Corollary 8.2 ([15, Proposition 2.12] and [41, Theorem 3.1]). Assume that $X$ is a Hilbert space and that $C$ is prox-regular at $\bar{x} \in C$. Then for some neighbourhood $U$ of $\bar{x}$ one has for all $x \in U \cap C$ and $y \in X$ that the directional derivatives of $P_{C}$ and $d_{C}$ at $x$ in the direction $y$ exist and

$$
\lim _{t \downarrow 0} \frac{1}{t}\left[P_{C}(x+t y)-x\right]=P_{K_{C}(x)}(y) \quad \text { and } d_{C}^{\prime}(x ; y)=d\left(y, K_{C}(x)\right) \text {. }
$$

\section{Convergence}

In this section we are interested in the behaviour of the projection mapping under convergence of prox-regular sets.

Let us begin by recalling some properties of the projection mapping of a proxregular set and by providing some additional facts. Consider a uniformly $r$-proxregular closed set $C$. We saw in the statement of Theorem 2.6 that the function 
$d_{C}^{2}$ is Fréchet differentiable on the $r$-open neighborhood $O_{C}(r)$ and that $P_{C}$ is a single-valued and norm-to-norm continuous mapping from $O_{C}(r)$ into $C$. Moreover, Proposition 5.5 in 9 gives for any $x \in O_{C}(r)$,

$$
D\left(\frac{1}{2} d_{C}^{2}\right)(x)=J\left(x-P_{C}(x)\right) .
$$

Concerning the continuity of $P_{C}$, Theorem 5.2 in $[9$ says more, in the sense that $P_{C}$ is locally Hölder continuous on $O_{C}(r)$. The following two propositions furnish some further points that we will need in Theorem 9.4 below. Recall that $q$ (resp. $s$ ) denotes the power type of the modulus of convexity (resp. smoothness) of the norm of $X$.

Proposition 9.1. Assume that the moduli of uniform convexity and smoothness of the norm $\|\cdot\|$ of $X$ are of power types $q$ and $s$, respectively. Let $r, r^{\prime}$ be two positive numbers with $r^{\prime}<\frac{r}{2}$ and let a number $\rho>0$. Then there exists some constant $\gamma \geq 0$ depending only on $r, r^{\prime}$ and $\rho$ such that, for any uniformly $r$-prox-regular closed subset $C$ of $X$, one has

$$
\left\|P_{C}\left(x_{1}\right)-P_{C}\left(x_{2}\right)\right\| \leq \gamma\left\|x_{1}-x_{2}\right\|^{\frac{1}{q}} \text { and }\left|D d_{C}^{2}\left(x_{1}\right)-D d_{C}^{2}\left(x_{2}\right)\right| \leq \gamma\left\|x_{1}-x_{2}\right\|^{\frac{s-1}{q}}
$$

for all $x_{1}, x_{2} \in O_{C}\left(r^{\prime}\right) \cap \rho \mathbb{B}$.

Proof. Take $\alpha \in] 0,1 / 2\left[\right.$ such that $r^{\prime}=\alpha r$. By Step 1 of the proof of Theorem 5.2 in [9] the truncated normal cone set-valued mapping $N_{C, r}^{P}(\cdot)$ is $J$-hypomonotone of degree 1 and hence so is the set-valued mapping $N_{C, \alpha r}^{P}(\cdot)$. Referring to the proof of Lemma 2.7 in [9] with $T(\cdot):=N_{C, \alpha r}^{P}(\cdot)$ and $\bar{r}:=1$, since $\alpha^{-1}>2 \bar{r}$ the development there reveals that, fixing $\lambda$ satisfying $2 \alpha<\lambda<1$, there exists some constant $\gamma^{\prime}>0$ depending only on $\alpha, \rho$, and $r$ such that $Q:=\left(I+\alpha J^{*} \circ N_{C, r}^{P}\right)^{-1}$ is on $\rho \mathbb{B} \cap \operatorname{Dom} Q$ a single-valued mapping for which

$$
\left\|Q\left(x_{1}\right)-Q\left(x_{2}\right)\right\| \leq \gamma^{\prime}\left\|x_{1}-x_{2}\right\|^{\frac{1}{q}} \quad \text { for all } x_{1}, x_{2} \in \rho \mathbb{B} \cap \operatorname{Dom} Q .
$$

Observe now that for $x \in O_{C}(\alpha r)$ we have $J\left(x-P_{C}(x)\right) \in N_{C}^{P}\left(P_{C}(x)\right.$ ) (by the definition of proximal normal functional) and $\left\|J\left(x-P_{C}(x)\right)\right\|<\alpha r$ and that those two facts imply that $J\left(x-P_{C}(x)\right) \in \alpha N_{C, r}^{P}\left(P_{C}(x)\right)$, that is, $P_{C}(x)=\left(I+\alpha J^{*}\right.$ 。 $\left.N_{C, r}^{P}\right)^{-1}(x)$. We may then reformulate (9.3) in the form

$$
\left\|P_{C}\left(x_{1}\right)-P_{C}\left(x_{2}\right)\right\| \leq \gamma^{\prime}\left\|x_{1}-x_{2}\right\|^{\frac{1}{q}} \quad \text { for all } x_{1}, x_{2} \in O_{C}\left(r^{\prime}\right) \cap \rho \mathbb{B} .
$$

Concerning the square distance function $d_{C}^{2}$, we know that it is (see (9.1) ) Fréchet differentiable on $O_{C}(r)$ with $D d_{C}^{2}(x)=2 J\left(x-P_{C}(x)\right)$ for all $x \in O_{C}(r)$. So using (2.9) and the latter inequality above, we have for all $x_{1}, x_{2} \in O_{C}\left(r^{\prime}\right) \cap \rho \mathbb{B}$,

$$
\begin{aligned}
\left\|D d_{C}^{2}\left(x_{1}\right)-D d_{C}^{2}\left(x_{2}\right)\right\| & \leq 2 L_{\rho}\left\|x_{1}-P_{C}\left(x_{1}\right)-x_{2}+P_{C}\left(x_{2}\right)\right\|^{s-1} \\
& \leq 2 L_{\rho}\left(\left\|x_{1}-x_{2}\right\|+\gamma^{\prime}\left\|x_{1}-x_{2}\right\|^{\frac{1}{q}}\right)^{s-1} \\
& \leq 2 L_{\rho}\left(\gamma^{\prime}+\left\|x_{1}-x_{2}\right\|^{1-\frac{1}{q}}\right)^{s-1}\left\|x_{1}-x_{2}\right\|^{\frac{s-1}{q}} \\
& \leq 2 L_{\rho}\left(\gamma^{\prime}+(2 \rho)^{1-\frac{1}{q}}\right)^{s-1}\left\|x_{1}-x_{2}\right\|^{\frac{s-1}{q}} .
\end{aligned}
$$

It suffices to take $\gamma=\max \left\{\gamma^{\prime}, 2 L_{\rho}\left(\gamma^{\prime}+(2 \rho)^{1-\frac{1}{q}}\right)^{s-1}\right\}$ to obtain both inequalities in the statement of the proposition.

In the proposition above, under the restriction $r^{\prime}<r / 2$, the power of Hölder continuity of $P_{C}$ over $O_{C}\left(r^{\prime}\right) \cap \rho \mathbb{B}$ is the constant $1 / q$ (the inverse of the power type 
of uniform convexity of $\|\cdot\|$ ); i.e., only the modulus $\gamma$ of Hölder continuity varies with $r^{\prime}$ and $\rho$. Relaxing the restriction $r^{\prime}<r / 2$ into $r^{\prime}<r$ and letting the power of Hölder continuity depend also on $r^{\prime}$ and $\rho$, we can prove the following result of Hölder continuity of $P_{C}$ on $O_{C}\left(r^{\prime}\right) \cap \rho \mathbb{B}$ but this time with any $r^{\prime}<r$.

Before stating the result, recall that for any $r>0$ the (closed) $r$-enlargement $C(r)$ of $C$ is given by $C(r):=\{x \in X: d(x, C) \leq r\}$.

Proposition 9.2. Assume that the moduli of uniform convexity and smoothness of the norm $\|\cdot\|$ of $X$ are of power types $q$ and $s$, respectively. Let $\rho, r, r^{\prime}$ be positive real numbers with $r^{\prime}<r$. Then there exist some positive constants $\gamma$ and $\theta \leq 1$ both depending only on $r^{\prime}, r$ and $\rho$, such that for any uniformly $r$-prox-regular closed set $C$ of $X$ one has

$\left\|P_{C}\left(x_{1}\right)-P_{C}\left(x_{2}\right)\right\| \leq \gamma\left\|x_{1}-x_{2}\right\|^{\theta}$ and $\left|D d_{C}^{2}\left(x_{1}\right)-D d_{C}^{2}\left(x_{2}\right)\right| \leq \gamma\left\|x_{1}-x_{2}\right\|^{\theta(s-1)}$ for all $x_{1}, x_{2} \in O_{C}\left(r^{\prime}\right) \cap \rho \mathbb{B}$.

More precisely, defining $\left(\alpha_{n}\right)_{n}$ by $\alpha_{1}=\frac{1}{2}$ and $\alpha_{n+1}=\frac{1+\alpha_{n}}{2}$, then for any $n \in \mathbb{N}$, $\left.\rho>0, \alpha^{\prime} \in\right] 0, \alpha_{n}[$, there exists some positive real number $k$ (depending only on $\rho$, $\alpha^{\prime}$ and $n$ ) such that, for any uniformly $r$-prox-regular closed set $C$ of $X$, the metric projection mapping $P_{C}$ is Hölder continuous on $O_{C}\left(\alpha^{\prime} r\right) \cap \rho \mathbb{B}$ with power $\theta:=\frac{1}{q^{n}}$ and with modulus $k$.

Proof. Let us call $\mathcal{P}(n)$ the property above in the second part of the proposition for $P_{C}$ of any uniformly $r$-prox-regular closed set $C$. Note that $\mathcal{P}(1)$ is Proposition 9.1. Suppose that $\mathcal{P}(n)$ is fulfilled and fix any uniformly $r$-prox-regular closed set $C$ and any $\left.\rho>0, \alpha^{\prime} \in\right] 0, \alpha_{n}\left[\right.$. We may suppose that $O_{C}(r) \cap \rho \mathbb{B} \neq \emptyset$. By Theorem 2.6 and Lemma 5.4 in [9], the closed set $C\left(\alpha^{\prime} r\right)$ is uniformly $\left(1-\alpha^{\prime}\right) r$-prox-regular. Then applying Proposition 9.1 to the set $C\left(\alpha^{\prime} r\right)$ yields that for any $\left.\lambda \in\right] 0,1[$,

$$
P_{C\left(\alpha^{\prime} r\right)} \text { is Hölder continuous on } O_{C\left(\alpha^{\prime} r\right)}\left(\lambda \frac{1-\alpha^{\prime}}{2} r\right) \cap \rho \mathbb{B} \text { with power } 1 / q
$$

and with modulus depending only on $\rho, \lambda, \alpha^{\prime}$, and $r$. On the other hand, we have that for any $t>0, O_{C}\left(\alpha^{\prime} r+t\right)=O_{C\left(\alpha^{\prime} r\right)}(t)$. Indeed, from Lemma 5.3(a) in 9] (see also [14]), for any $\tau>0$ and any $u \notin C(\tau)$ one has $d_{C}(u)=\tau+d_{C(\tau)}(u)$. Hence, for any $u \in X$, one has the equivalence $d_{C}(u)<\alpha^{\prime} r+t \Leftrightarrow d_{C\left(\alpha^{\prime} r\right)}(u)<t$; that is, the desired equality holds. This entails that

$$
O_{C\left(\alpha^{\prime} r\right)}\left(\lambda \frac{1-\alpha^{\prime}}{2} r\right)=O_{C}\left(\alpha^{\prime} r+\lambda \frac{1-\alpha^{\prime}}{2} r\right) .
$$

By (9.5) and (9.6) there exists some constant $K_{1}>0$ (depending only on $\rho, \lambda, \alpha^{\prime}$, and $r$ ) such that

$$
\left\|P_{C\left(\alpha^{\prime} r\right)}\left(u_{1}\right)-P_{C\left(\alpha^{\prime} r\right)}\left(u_{2}\right)\right\| \leq K_{1}\left\|u_{1}-u_{2}\right\|^{1 / q} \text { for all } u_{i} \in O_{C}\left(\alpha^{\prime} r+\lambda \frac{1-\alpha^{\prime}}{2} r\right) .
$$

Fixing $b_{\rho} \in O_{C}(r) \cap \rho \mathbb{B} \neq \emptyset$, we find some $a_{\rho} \in C$ with $\left\|a_{\rho}\right\| \leq r+\rho$. Combining this with (9.7), we obtain that there is some $\rho^{\prime}>0$ (depending only on $\rho, \lambda, \alpha^{\prime}$, and $r$ ) such that

$$
\left\|P_{C\left(\alpha^{\prime} r\right)}(u)\right\| \leq \rho^{\prime} \quad \text { for all } u \in(\rho \mathbb{B}) \cap O_{C}\left(\alpha^{\prime} r+\lambda \frac{1-\alpha^{\prime}}{2} r\right)
$$

(take $u_{1}=u$ and $u_{2}=a_{\rho}$ in (9.7)). The equality (9.6) also implies, according to Theorem 2.6. that for any $u \in O_{C}\left(\alpha^{\prime} r+\lambda \frac{1-\alpha^{\prime}}{2} r\right) \backslash C\left(\alpha^{\prime} r\right)$, the points $y:=$ 
$P_{C\left(\alpha^{\prime} r\right)}(u)$ and $z:=P_{C}(y)$ exist. By (c) of Lemma 5.3 in 9 , for any such $u$ we have consequently $z=P_{C}(u)$ and so, $P_{C}(u)=P_{C} \circ P_{C\left(\alpha^{\prime} r\right)}(u)$. From this, if $u_{1}, u_{2} \in(\rho \mathbb{B}) \cap\left(O_{C}\left(\alpha^{\prime} r+\lambda \frac{1-\alpha^{\prime}}{2} r\right) \backslash C\left(\alpha^{\prime} r\right)\right)$, then for any $\left.\alpha^{\prime \prime} \in\right] \alpha^{\prime}, \alpha_{n}[$ we have

$$
\begin{aligned}
\left\|P_{C}\left(u_{1}\right)-P_{C}\left(u_{2}\right)\right\| & =\left\|P_{C}\left(P_{C\left(\alpha^{\prime} r\right)}\left(u_{1}\right)\right)-P_{C}\left(P_{C\left(\alpha^{\prime} r\right)}\left(u_{2}\right)\right)\right\| \\
& \leq K_{2}\left\|P_{C\left(\alpha^{\prime} r\right)}\left(u_{1}\right)-P_{C\left(\alpha^{\prime} r\right)}\left(u_{2}\right)\right\|^{1 / q^{n}}
\end{aligned}
$$

for some constant $K_{2}>0$ (depending only on $\rho^{\prime}, \alpha^{\prime \prime}$, and $n$ ), according to $\mathcal{P}(n)$ and to the fact that, by (9.8), for $i=1,2, P_{C\left(\alpha^{\prime} r\right)}\left(u_{i}\right) \in \rho^{\prime} \mathbb{B} \cap O_{C}\left(\alpha^{\prime \prime} r\right)$. Taking (9.7) into account we obtain that there exists some constant $K_{3}$ (depending only on $\rho, \lambda, \alpha^{\prime}, r$ and $\left.n\right)$ such that for all $u_{1}, u_{2} \in(\rho \mathbb{B}) \cap\left(O_{C}\left(\alpha^{\prime} r+\lambda \frac{1-\alpha^{\prime}}{2} r\right) \backslash C\left(\alpha^{\prime} r\right)\right)$,

$$
\left\|P_{C}\left(u_{1}\right)-P_{C}\left(u_{2}\right)\right\| \leq K_{3}\left\|u_{1}-u_{2}\right\|^{1 / q^{n+1}},
$$

and this inequality is still true for all $u_{i} \in \rho \mathbb{B}$ satisfying $\alpha^{\prime} r \leq d\left(u_{i}, C\right)<\alpha^{\prime} r+$ $\lambda \frac{1-\alpha^{\prime}}{2} r$. On $\rho \mathbb{B} \cap C\left(\alpha^{\prime} r\right), \mathcal{P}(n)$ ensures that $P_{C}$ also has the Hölder continuity property with power $1 / q^{n}$ and hence also with power $1 / q^{n+1}$ (because of the boundedness of $\rho \mathbb{B} \cap C\left(\alpha^{\prime} r\right)$ ). Then there exists some positive constant $K_{4}$ (depending only on $\rho, \lambda, \alpha^{\prime}, r$ and $\left.n\right)$ such that

$$
\left\|P_{C}\left(u_{1}\right)-P_{C}\left(u_{2}\right)\right\| \leq\left(K_{4} / 2\right)\left\|u_{1}-u_{2}\right\|^{1 / q^{n+1}}
$$

for all $u_{1}, u_{2} \in(\rho \mathbb{B}) \cap O_{C}\left(\alpha^{\prime} r+\lambda \frac{1-\alpha^{\prime}}{2} r\right)$ satisfying either $d\left(u_{i}, C\right) \geq \alpha^{\prime} r$ for $i=1$ and $i=2$ or $d\left(u_{i}, C\right) \leq \alpha^{\prime} r$ for $i=1$ and $i=2$. In the remaining case where $u_{1}, u_{2} \in(\rho \mathbb{B}) \cap O_{C}\left(\alpha^{\prime} r+\lambda \frac{1-\alpha^{\prime}}{2} r\right)$ with $d\left(u_{1}, C\right)<\alpha^{\prime} r$ and $d\left(u_{2}, C\right)>\alpha^{\prime} r$, the continuity of $d(\cdot, C)$ over the line segment $\left[u_{1}, u_{2}\right]$ yields some point $\left.u_{0} \in\right] u_{1}, u_{2}[$ such that $d\left(u_{0}, C\right)=\alpha^{\prime} r$; then

$$
\left\|P_{C}\left(u_{1}\right)-P_{C}\left(u_{2}\right)\right\| \leq\left(K_{4} / 2\right)\left(\left\|u_{1}-u_{0}\right\|^{1 / q^{n+1}}+\left\|u_{0}-u_{2}\right\|^{1 / q^{n+1}}\right) \leq K_{4}\left(\left\|u_{1}-u_{2}\right\|^{1 / q^{n+1}},\right.
$$

the second inequality being due to the inequalities $\left\|u_{i}-u_{0}\right\| \leq\left\|u_{1}-u_{2}\right\|$. The mapping $P_{C}$ is then Hölder continuous on $\rho \mathbb{B} \cap O_{C}\left(\alpha^{\prime} r+\lambda \frac{1-\alpha^{\prime} r}{2} r\right)$ with modulus $K_{4}$ and power $1 / q^{n+1}$.

We have shown that, for any $\lambda \in] 0,1\left[, \alpha^{\prime} \in\right] 0, \alpha_{n}[$, and $\rho>0$, there exists some positive real number $k$ (depending only on $\rho, \alpha^{\prime}, \lambda$, and $n$ ) such that, for any uniformly $r$-prox-regular set $C$, the metric projection mapping $P_{C}$ is Hölder continuous on $\rho \mathbb{B} \cap O_{C}\left(\alpha^{\prime} r+\lambda \frac{1-\alpha^{\prime}}{2} r\right)$ with power $1 / q^{n+1}$ and with modulus $k$. This means that for any $\left.\alpha^{\prime} \in\right] 0, \alpha_{n}\left[\right.$, there is some positive $k$ (depending only on $\rho, \alpha^{\prime}$, and $n$ ) such that, for any uniformly $r$-prox-regular set $C$ of $X$, the mapping $P_{C}$ is Hölder continuous on $\rho \mathbb{B} \cap O_{C}\left(\alpha^{\prime} r+\frac{1-\alpha^{\prime}}{2} r\right)$ with power $1 / q^{n+1}$ and modulus $k$. Consequently $\mathcal{P}(n+1)$ holds true since $\alpha^{\prime} r+\frac{1-\alpha^{\prime}}{2} r=\frac{1+\alpha^{\prime}}{2} r$.

The Hölder continuity of the function $D d_{C}^{2}(\cdot)$ is obtained as in the proof of Proposition 9.1 .

Remark 9.3. When $(X,\|\cdot\|)$ is a Hilbert space, where $\|\cdot\|$ is the norm associated with the inner product, the behaviour of $P_{C}(\cdot)$ and $D d_{C}^{2}(\cdot)$ is distinctly better. Indeed, by [39, for any real positive numbers $r, r^{\prime}$ with $r^{\prime}<r$ and any uniformly $r$-prox-regular closed set $C$ of $X$ one has

$$
\left\|P_{C}\left(x_{1}\right)-P_{C}\left(x_{2}\right)\right\| \leq \frac{r}{r-r^{\prime}}\left\|x_{1}-x_{2}\right\|
$$


for all $x_{1}, x_{2} \in O_{C}\left(r^{\prime}\right)$, and hence

$$
\left\|D d_{C}^{2}\left(x_{1}\right)-D d_{C}^{2}\left(x_{2}\right)\right\| \leq 2\left(1+\frac{r}{r-r^{\prime}}\right)\left\|x_{1}-x_{2}\right\|
$$

according to (9.1) and to the Lipschitz property of $J$ here with Lipschitz modulus 1. So, not only the Lipschitz continuity (instead of the Hölder one) is available for the metric projection but also no restriction to bounded subsets of $O_{C}\left(r^{\prime}\right)$ is required.

We can now study convergence properties of families of uniformly prox-regular sets. Let $T \cup\left\{t_{0}\right\}$ be a topological space with $t_{0}$ as a cluster point of $T$. Recall that a family $\left(C_{t}\right)_{t \in T}$ of nonempty closed subsets of $X$ converges in the sense of Attouch-Wets (see [1, 4, 40]) to a closed subset $C$ of $X$ when $t$ goes to $t_{0}$ provided that for each positive real number $\rho$ one has

$$
\sup _{x \in \rho \mathbb{B}}\left|d_{C_{t}}(x)-d_{C}(x)\right| \underset{t \rightarrow t_{0}}{\longrightarrow} 0 .
$$

Theorem 9.4. Assume that the moduli of uniform convexity and smoothness of the norm $\|\cdot\|$ of $X$ are of power type. Let $\left(C_{t}\right)_{t \in T}$ be a family of closed uniformly $r$-prox-regular sets of $X$ which converges in the sense of Attouch-Wets to a closed set $C$ of $X$. Then $C$ is uniformly $r$-prox-regular and for each $x_{0} \in X$ satisfying $d\left(x_{0}, C\right)<r$ the mapping $t \mapsto P_{C_{t}}\left(x_{0}\right)$ and the function $t \mapsto D d_{C_{t}}^{2}\left(x_{0}\right)$ are defined on a neighbourhood of $t_{0}$ and

$$
P_{C_{t}}\left(x_{0}\right) \underset{t \rightarrow t_{0}}{\longrightarrow} P_{C}\left(x_{0}\right) \quad \text { and } \quad D d_{C_{t}}^{2}\left(x_{0}\right) \underset{t \rightarrow t_{0}}{\longrightarrow} D d_{C}^{2}\left(x_{0}\right) .
$$

Proof. Fix a positive number $r^{\prime}$ with $d\left(x_{0}, C\right)<r^{\prime}<r$ and choose by (9.10) some neighbourhood $T_{0}^{\prime}$ of $t_{0}$ and some $\beta>0$ such that $d\left(x, C_{t}\right)<r^{\prime}$ for all $t \in T_{0}^{\prime} \backslash\left\{t_{0}\right\}$ and $x \in x_{0}+2 \beta \mathbb{B}$. According to Proposition 9.2 there exist some positive constants $\gamma$ and $\sigma \leq 1$ (both depending only $x_{0}, \beta, r^{\prime}$ and $r$ ) such that for all $t \in T_{0}^{\prime}$ with $t \neq t_{0}$ and $x_{1}, x_{2} \in x_{0}+2 \rho \mathbb{B}$ the derivatives $D d_{C_{t}}^{2}\left(x_{i}\right), i=1,2$, exist and

$$
\left\|D d_{C_{t}}^{2}\left(x_{1}\right)-D d_{C_{t}}^{2}\left(x_{2}\right)\right\| \leq \gamma\left\|x_{1}-x_{2}\right\|^{\sigma} .
$$

Fix another neighbourhood $T_{0}^{\prime \prime}$ of $t_{0}$ with $T_{0}^{\prime \prime} \subset T_{0}^{\prime}$ and such that, according to (9.10), the function $v$ given for $\rho:=1+2 \beta+\left\|x_{0}\right\|$ by

$$
v(t):=\sup _{x \in \rho \mathbb{B}}\left|d_{C_{t}}(x)-d_{C}(x)\right|
$$

is bounded on $T_{0}:=T_{0}^{\prime \prime} \backslash\left\{t_{0}\right\}$. Writing

$d_{C_{t}}(x) \leq d_{C_{t}}\left(x_{0}\right)+\left\|x-x_{0}\right\| \leq d_{C}\left(x_{0}\right)+\left\|x-x_{0}\right\|+v(t) \quad$ for all $x \in X$ and $t \in T$, we see that there exists some constant $M>0$ such that

$$
d_{C_{t}}(x) \leq M / 2 \text { for all }(t, x) \in T_{0} \times \rho \mathbb{B} .
$$

Now fix any positive number $\varepsilon<\beta$. Choose by (9.10) a neighbourhood $T^{\prime}{ }_{\varepsilon} \subset T_{0}^{\prime \prime}$ of $t_{0}$ such that for $T_{\varepsilon}:=T_{\varepsilon}^{\prime} \backslash\left\{t_{0}\right\}$,

$$
\left|d_{C_{t}}(x)-d_{C}(x)\right| \leq \frac{\varepsilon^{\sigma+1}}{2} \text { for all } t \in T_{\varepsilon} \text { and } x \in \rho \mathbb{B} .
$$

Fix any $x \in B\left(x_{0}, \beta\right)$, any $w \in X$ with $\|w\|=\varepsilon$, and any $t, \tau \in T_{\varepsilon}$. We have

$$
d_{C_{t}}^{2}(x+w)-d_{C}^{2}(x)=\left\langle D d_{C_{t}}^{2}(x), w\right\rangle+\int_{0}^{1}\left\langle D d_{C_{t}}^{2}(x+s w)-D d_{C_{t}}^{2}(x), w\right\rangle d s
$$


and a similar equality with $d_{C_{\tau}}^{2}$ in place of $d_{C_{t}}^{2}$. Hence

$$
\begin{aligned}
& \left\langle D d_{C_{t}}^{2}(x)-D d_{C_{2}}^{2}(x), w\right\rangle \\
= & {\left[d_{C_{t}}^{2}(x+w)-d_{C_{\tau}}^{2}(x+w)\right]-\left[d_{C_{t}}^{2}(x)-d_{C_{\tau}}^{2}(x)\right] } \\
& -\int_{0}^{1}\left\langle D d_{C_{t}}^{2}(x+s w)-D_{C_{t}}^{2}(x), w\right\rangle+\int_{0}^{1}\left\langle D d_{C_{\tau}}^{2}(x+s w)-D d_{C_{\tau}}^{2}(x), w\right\rangle .
\end{aligned}
$$

Further, on the one hand by (9.12) we have

$$
\begin{aligned}
\left|\int_{0}^{1}\left\langle D d_{C_{t}}^{2}(x+s w)-D d_{C_{t}}^{2}(x), w\right\rangle d s\right| & \leq \int_{0}^{1} \gamma s^{\sigma}\|w\|^{\sigma+1} d s=\frac{1}{\sigma+1} \gamma\|w\|^{\sigma+1} \\
& \leq \gamma \varepsilon^{\sigma+1}
\end{aligned}
$$

and a similar inequality with $d_{C_{\tau}}^{2}$ in place of $d_{C_{t}}^{2}$. On the other hand by (9.13) and (9.14), with $y:=x+w$ or $y:=x$ we also have

$$
\left|d_{C_{t}}^{2}(y)-d_{C_{\tau}}^{2}(y)\right|=\left(d_{C_{t}}(y)+d_{C_{\tau}}(y)\right)\left|d_{C_{t}}(y)-d_{C_{\tau}}(y)\right| \leq M \varepsilon^{\sigma+1} .
$$

Consequently

$$
\left\langle D d_{C_{t}}^{2}(x)-D d_{C_{\tau}}^{2}(x), w\right\rangle \leq(2 M+2 \gamma) \varepsilon^{\sigma+1}
$$

and hence for every $u$ in $X$ with $\|u\|=1$,

$$
\left\langle D d_{C_{t}}^{2}(x)-D d_{C_{\tau}}^{2}(x), u\right\rangle \leq(2 M+2 \gamma) \varepsilon^{\sigma},
$$

which ensures that

$$
\left\|D d_{C_{t}}^{2}(x)-D d_{C_{\tau}}^{2}(x)\right\| \leq(2 M+2 \gamma) \varepsilon^{\sigma} .
$$

This uniform Cauchy property says that the family $\left(D d_{C_{t}}^{2}\right)_{t}$ converges uniformly to some mapping from $B\left(x_{0}, \beta\right)$ into $X^{*}$. This is known to entail that the function $d_{C}^{2}$ is Fréchet differentiable on $B\left(x_{0}, \beta\right)$ and hence, in particular, Fréchet differentiable at $x_{0}$ with

$$
D d_{C_{t}}^{2}\left(x_{0}\right) \underset{t \rightarrow t_{0}}{\longrightarrow} D d_{C}^{2}\left(x_{0}\right) .
$$

Then the function $d_{C}$ is Fréchet differentiable on the open tube $U_{C}(r)$ and this differentiability property translates through Theorem 2.6 the uniform $r$-prox-regularity of $C$. Further (9.1) and (9.15) yield

$$
P_{C_{t}}\left(x_{0}\right) \underset{t \rightarrow t_{0}}{\longrightarrow} P_{C}\left(x_{0}\right) \text {. }
$$

This completes the proof of the proposition.

\section{REFERENCES}

[1] H. Attouch and R. J.-B. Wets, Isometries for the Legendre-Fenchel transform, Trans. Amer. Math. Soc. 296 (1986), 33-60. MR837797 (87k:49023)

[2] D. Aussel, A. Daniilidis and L. Thibault, Subsmooth sets: Functional characterizations and related concepts, Trans. Amer. Math. Soc. 357 (2004), 1275-1301. MR.2115366 (2007b:49033)

[3] B. Beauzamy, Introduction to Banach spaces and their geometry, 2nd edition, North-Holland, Amsterdam (1985). MR889253 (88f:46021)

[4] G. Beer, Topologies on Closed and Closed Convex Sets, Kluwer Academic Publishers, NorthHolland, Dordrecht (1993). MR1269778 (95k:49001)

[5] F. Bernard and L. Thibault, Prox-regular functions in Hilbert spaces, J. Math. Anal. Appl. 303 (2005), 1-14. MR2113863(2005h:49043)

[6] F. Bernard and L. Thibault, Prox-regular functions and sets in Banach spaces, Set-Valued Analysis 12 (2004), 25-47. MR2069350 (2005c:49034)

[7] F. Bernard and L. Thibault, Uniform prox-regularity of functions and epigraphs in Hilbert spaces, Nonlinear Anal. 60 (2005), 187-207. MR2101873 (2005h:49035) 
[8] F. Bernard, L. Thibault and D. Zagrodny, Integration of primal lower nice functions in Hilbert spaces, J. Optimization Theory Appl. 124 (2005), 561-579. MR2129814 (2005k:49046)

[9] F. Bernard, L. Thibault and N. Zlateva, Characterizations of prox-regular sets in uniformly convex Banach spaces, J. Convex Analysis 13 (2006), 525-559. MR2291551 (2008f:49027)

[10] J. M. Borwein and J. R. Giles, The proximal normal formula in Banach space, Trans. Amer. Math. Soc. 302 (1987), 371-381. MR887515 (88m:49013)

[11] J. M. Borwein and H. M. Strójwas, Tangential approximations, Nonlinear Anal. 9 (1985), 1347-1366. MR820646 (87i:90320)

[12] J. M. Borwein and H. M. Strójwas, Proximal analysis and boundaries of closed sets in Banach space. I. Theory, Canad. J. Math. 38 (1986), 431-452. MR833578(87h:90258)

[13] J. M. Borwein and H. M. Strójwas, Proximal analysis and boundaries of closed sets in Banach space. II. Applications, Canad. J. Math. 39 (1987), no. 2, 428-472. MR899844 (88f:46034)

[14] M. Bounkhel and L. Thibault, On various notions of regularity of sets in nonsmooth analysis, Nonlinear Anal. 48 (2002), 223-246. MR.1870754 (2002j:46048)

[15] A. Canino, On p-convex sets and geodesics, J. Differential Equations 75 (1988), 118-157. MR.957011 (90b:58044)

[16] F. H. Clarke, Optimization and nonsmooth analysis, John Wiley \& Sons, New York (1983). MR.709590 (85m:49002)

[17] F. H. Clarke, Yu. S. Ledyaev, J. R. Stern and R. P. Wolenski, Nonsmooth Analysis and Control Theory, Springer, New York (1998). MR1488695 (99a:49001)

[18] F. H. Clarke, J. R. Stern and R. P. Wolenski, Proximal smoothness and the lower- $C^{2}$ property, J. Convex Analysis 2 (1995), 117-144. MR1363364 (96j:49014)

[19] G. Colombo and V. V. Goncharov, Variational inequalities and regularity properties of sets in Hilbert spaces, J. Convex Analysis 8 (2001), 197-221. MR 1829062 (2002f:49031)

[20] R. Correa, A. Jofre and L.Thibault, Subdifferential monotonicity as characterization of convex functions, Numer. Funct. Anal. Optimiz. 15 (1994), 531-536. MR.1281560 (95d:49029)

[21] M. Degiovanni, A. Marino and M. Tosques, General properties of $(p, q)$-convex functions and operators, Ricerche Mat. 32 (1983), 285-319. MR766683 (86j:49035)

[22] R. Deville, G. Godefroy and V. Zizler, Smoothness and Renormings in Banach Spaces, Pitman Monographs and Surveys in Pure and Applied Mathematics, 64, John Wiley \& Sons Inc., New York (1993). MR.1211634 (94d:46012)

[23] J. Diestel, Geometry of Banach spaces - selected topics. Lecture Notes in Mathematics, vol. 485, Springer-Verlag, Berlin-New York (1975). MR0461094 (57:1079)

[24] J. F. Edmond and L. Thibault, BV solutions of nonconvex sweeping process differential inclusion with perturbation, J. Differential Equations 226 (2006), 135-179. MR.2232433 (2008a:34022)

[25] M. Fabian, P. Habala, P. Hájek, V. Montesinos Santalucía, J. Pelant and V. Zizler, Functional Analysis and Infinite-Dimensional Geometry (CMS books in mathematics), Springer-Verlag, New york (2001). MR1831176 (2002f:46001)

[26] H. Federer, Curvature measures, Trans. Amer. Math. Soc. 93 (1959), 418-491. MR0110078 $(22: 961)$

[27] A. D. Ioffe, Proximal analysis and approximate subdifferentials, J. London Math. Soc. 41 (1990), 175-192. MR.1063554 (91i:46045)

[28] A. D. Ioffe, Metric regularity and subdifferential calculus, Russian Math. Surveys 55 (2000), 501-558. MR1777352 (2001j:90002)

[29] M. Ivanov and N. Zlateva, On primal lower nice property, C.R. Acad. Bulgare Sci. 54 (11) (2001), 5-10. MR 1878038 (2002k:49035)

[30] A. Jourani and L. Thibault, Metric regularity for strongly compactly Lipschitzian mappings, Nonlinear Analysis 24 (1995), 229-240. MR1312592 (95j:49017)

[31] K. S. Lau, Almost Chebyshev subsets in reflexive Banach spaces, Indiana Univ. Math. J. 27 (1978), no. 5, 791-795. MR0510772 (58:23286)

[32] A. B. Levy, R. A. Poliquin and L. Thibault, Partial extension of Attouch's theorem with applications to proto-derivatives on subgradient mappings, Trans. Amer. Math. Soc. 347 (1995), 1269-1294. MR.1290725 (95k:49035)

[33] S. Marcellin and L. Thibault, Evolution problems associated with primal lower nice functions, J. Convex Analysis 13 (2006), 385-421. MR2252239(2007e:49029)

[34] B. Maury and J. Venel, Un modèle de mouvements de foule, ESAIM Proceedings 18 (2007), 143-152. MR2404902 (2009f:90046) 
[35] B. S. Mordukhovich, Variational Analysis and Generalized Differentiation I and II, Springer, New York, Comprehensive Studies in Mathematics, Vols. 330 and 331 (2005). MR2191744 (2007b:49003a) MR 2191745 (2007b:49003b)

[36] B. S. Mordukhovich and Y. Shao, Nonsmooth sequential analysis in Asplund spaces, Trans. Amer. Math. Soc. 124 (1996), 1235-1280. MR1333396 (96h:49036)

[37] R. A. Poliquin, Integration of subdifferentials of nonconvex functions, Nonlinear Anal. 17 (1991), 385-398. MR.1123210 (92j:49008)

[38] R. A. Poliquin and R. T. Rockafellar, Prox-regular functions in variational analysis, Trans. Amer. Math. Soc. 348 (1996), 1805-1838. MR1333397 (96h:49039)

[39] R. A. Poliquin, R. T. Rockafellar and L. Thibault, Local differentiability of distance functions, Trans. Amer. Math. Soc. 352 (2000), 5231-5249. MR1694378 (2001b:49024)

[40] R. T. Rockafellar and R. J.-B. Wets, Variational Analysis, Springer-Verlag, Berlin, Comprehensive Studies in Mathematics, vol. 317 (1998). MR.1491362 (98m:49001)

[41] A. S. Shapiro, Existence and differentiability of metric projections in Hilbert spaces, SIAM J. Optimization 4 (1994), 130-141. MR1260410 (94m:90111)

[42] L. Thibault, Sweeping process with regular and nonregular sets, J. Differential Equations 193 (2003), 1-26. MR.1994056 (2004c:34040)

[43] L. Thibault, Regularization of nonconvex sweeping process in Hilbert space, Set-Valued Anal. 16 (2008), 319-333. MR2399209 (2009c:34020)

[44] L. Thibault and D. Zagrodny, Integration of subdifferentials of lower semicontinuous functions on Banach spaces, J. Math. Anal. Appl. 189 (1995), 33-58. MR.1312029 (95i:49032)

[45] Z. B. Xu and G. F. Roach, Characteristic inequalities of uniformly convex and uniformly smooth Banach spaces, J. Math. Anal. Appl. 157 (1991), 189-210. MR1109451 (92i:46023)

[46] E. H. Zarantonello, Projections on convex sets in Hilbert space and spectral theory I and II. In E. H. Zarantonello, ed., Contributions to Nonlinear Functional Analysis, Academic Press, New York (1971), pp. 237-424. MR0388177 (52:9014) MR0388178 (52:9015)

Département de Mathématiques, Université Montpellier II, CC 051, Place Eugène Bataillon, 34095 Montpellier cedex 5, France

E-mail address: bernard@math.univ-montp2.fr

Département de Mathématiques, Université Montpellier II, CC 051, Place Eugène Bataillon, 34095 Montpellier Cedex 5, France

E-mail address: thibault@math.univ-montp2.fr

Department of Mathematics and Informatics, Sofia University, 5 James Bourchier Blvd., 1164 Sofia, Bulgaria

E-mail address: zlateva@fmi.uni-sofia.bg 